\title{
Restituer les bidonvilles de Nanterre : l'apport d'un outil de visualisation 3D à un projet de sciences sociales
}

Restoring the Shantytowns of Nanterre: Benefits of a 3D Visualisation Tool for a Social Sciences Project

Paul Lecat, Émile Blettery, Lætitia Delavoipière, Frédéric Saly-Giocanti, Sylvaine Conord, Valérie Gouet-Brunet, Alexandre Devaux, Mathieu Brédif et Frédéric Moret

\section{OpenEdition Journals}

Édition électronique

URL : https://journals.openedition.org/revuehn/1946

DOI : 10.4000/revuehn.1946

ISSN : 2736-2337

Éditeur

Humanistica

\section{Référence électronique}

Paul Lecat, Émile Blettery, Lætitia Delavoipière, Frédéric Saly-Giocanti, Sylvaine Conord, Valérie GouetBrunet, Alexandre Devaux, Mathieu Brédif et Frédéric Moret, « Restituer les bidonvilles de Nanterre l'apport d'un outil de visualisation 3D à un projet de sciences sociales ", Humanités numériques [En ligne], 3 | 2021, mis en ligne le 01 mai 2021, consulté le 16 juillet 2021. URL : http:// journals.openedition.org/revuehn/1946; DOI : https://doi.org/10.4000/revuehn.1946

Les contenus de la revue Humanités numériques sont mis à disposition selon les termes de la Licence Creative Commons Attribution 4.0 International. 


\title{
humanités numériques
}

$3 \mid 2021$

Humanités numériques spatialisées

INTÉGRATION ET USAGES DES DONNÉES HISTORIQUES ET PATRIMONIALES

\section{Restituer les bidonvilles de Nanterre : l'apport d'un outil de visualisation 3D à un projet de sciences sociales}

Restoring the Shantytowns of Nanterre: Benefits of a $3 D$ Visualisation Tool for a Social Sciences Project

\author{
Paul Lecat, Émile Blettery, Lætitia Delavoipière, Frédéric \\ Saly-Giocanti, Sylvaine Conord, Valérie Gouet-Brunet, \\ Alexandre Devaux, Mathieu Brédif et Frédéric Moret
}

\section{Résumés}

$\mathrm{Au}$ milieu des années 1950 surgissent à Nanterre les premières cabanes de fortune abritant des travailleurs algériens. Bientôt, ces baraques informelles s'agrègent et finissent par former des ensembles urbains, présentés et administrés comme des bidonvilles, et la ville de Nanterre y est alors durablement associée. Cet article se propose de revenir sur une expérience de recherche interdisciplinaire autour de cet objet d'étude. Des chercheurs en histoire et en sociologie urbaine ont collaboré avec des informaticiens de l'IGN afin d'utiliser et d'enrichir une plateforme de spatialisation et de visualisation de données hétérogènes pour documenter l'histoire de ces bidonvilles et comprendre la formation et la permanence de ces lieux dans la mémoire collective actuelle.

In the mid-1950s, the first improvised huts for Algerian workers appeared in Nanterre. Soon, these informal shacks grew together and ended up forming urban complexes, presented and administered as shantytowns, and the city of Nanterre was then durably associated with them. This article proposes to review an interdisciplinary research experience around this object of study. Researchers in urban history and sociology collaborated with computer scientists from the IGN in order to use and enrich a platform for spatialisation and visualisation of hetero- 
geneous data to document the history of these shantytowns and to understand the formation and permanence of these places in the current collective memory.

\section{Entrées d'index}

MOTS-CLÉS : humanités numériques spatialisées, histoire, système d'information géographique, sociologie, imagerie, analyse spatiale

KEYWORDS: spatial digital humanities, geographic information system, history, sociology, image processing, spatial analysis

Dans les années 1950, alors que la France se reconstruit progressivement, les taudis et les bidonvilles s'étendent dans les périphéries des villes, en particulier dans l'agglomération parisienne. Cette nouvelle " crise du logement " (Fourcaut et Voldman 2013) et la " question des bidonvilles " (Blanc-Chaléard 2016) s'imposent dans le débat public, surtout après l'appel de l'abbé Pierre lors de l'hiver 1954. En parallèle de vastes opérations urbaines modernes, une autre ville se construit donc, particulièrement précaire et caractérisée par une mobilité et une fluidité intense. Les bidonvilles de Nanterre, qui se forment au milieu des années 1950, s'imposent rapidement dans les esprits comme le symbole de cet habitat délabré, cristallisent de ce fait toutes les représentations et attirent le regard des observateurs sociaux et de l'administration. Pour autant, le moment et les raisons de la formation de ces bidonvilles demeurent méconnus. De même, la matérialité concrète de ces ensembles urbains s'efface souvent devant les quelques photographies misérabilistes qui restent bien gravées dans les mémoires. Du fait de leur nature, les logements informels de ces bidonvilles laissent effectivement peu de documentation archivistique au moment de leur formation et disparaissent ensuite sans laisser de traces matérielles. Il n'est effectivement pas nécessaire de déposer un permis de construire ou de procéder à l'achat d'un terrain devant un notaire pour élever sa baraque sur un terrain vague de Nanterre. Les sources concernant ces formes urbaines existent pourtant : les bidonvilles ont été largement investis par le milieu associatif et les pouvoirs publics, qui ont produit une abondante documentation peu exploitable car elle est éclatée au sein de nombreux lieux de conservation avec de fortes difficultés d'accès. En particulier, deux types d'archives ont retenu notre attention pour aborder ces problématiques : d'une part, le recensement de 1954 du département de la Seine à l'échelle de l'îlot livre des données sur la morphologie sociale et spatiale de la ville de Nanterre, notamment sur la situation du logement ; d'autre part, les fonds de photographies aériennes permettent d'appréhender la matérialité de ces constructions et d'intégrer une vision diachronique des changements urbains de la commune. Pour exploiter ces sources et composer ce corpus de recherche, il convient donc de procéder au traitement et au croisement de ces données de natures très diverses qui n'ont pour seul point commun qu'un certain ancrage spatial. Évidemment, le classique système d'information géographique (SIG) s'est imposé dans un premier temps, en particulier pour l'exploita- 
tion du recensement. Cependant, le besoin d'un outil de traitement et de visualisation plus poussé nous a conduits à nous emparer de la plateforme de l'IGN iTowns. Cette plateforme est un moteur de restitution permettant une navigation spatiotemporelle, immersive et interactive dans un environnement tridimensionnel qui ne demande qu'à être enrichi de fonds iconographiques anciens avant d'être dévoilé au grand public. Dans le cadre de cette étude sur les bidonvilles de Nanterre, cet outil a permis de réunir l'ensemble de la documentation utilisée pour permettre d'éclairer les phénomènes de constitution de ces formes urbaines si particulières et leur persistance dans les mémoires collectives actuelles.

Ces premiers travaux de recherche ont aussi une valeur de test et visent à valider une méthodologie qui permettrait d'aborder bien d'autres problématiques liées au développement de l'agglomération parisienne au milieu du $\mathrm{xx}^{\mathrm{e}}$ siècle. Effectivement, les sources et les données utilisées concernent l'ensemble du Grand Paris et ouvrent de nombreuses opportunités de recherche ${ }^{1}$. Cet article se propose donc de revenir sur les méthodes permettant la constitution de ce corpus de sources, puis de présenter la plateforme iTowns et son utilité pour des recherches en sciences sociales, ainsi que les premiers résultats de cette enquête.

\section{Les données spatiales}

Deux types d'archives ont été mobilisés pour mieux comprendre ces phénomènes urbains. D'une part, le recensement de $1954^{2}$ a fait l'objet d'un lourd travail de saisie avec la constitution d'un SIG. En parallèle, les fonds photographiques de l'IGN ainsi que les images des archives départementales des Hauts-de-Seine (AD92, utilisées dans le programme $A L E$ GORIA) géoréférencés au sein d'un contexte 3D dans iTowns ${ }^{3}$. 


\section{Spatialiser le recensement de 1954}

Le projet Seine 54 a été lancé au cours de l'année 2019 suite à la découverte d'une source statistique sans équivalent. En mai 1954, quelques mois après le vibrant appel de l'abbé Pierre, la France organise une vaste opération de recensement. Ce recensement général de la population a laissé comme archives les fiches nominatives de chaque famille du pays, encore inaccessibles au public, ainsi que des synthèses présentées sous forme de tableaux agrégés à plusieurs échelles administratives (le pays, les régions, les départements, etc.). Ces sources classiques qui permettent l'étude de la répartition par âge ou par composition socioprofessionnelle offrent un aperçu synthétique de la population française, mais n'autorisent que peu d'analyses fines spatialisées des phénomènes sociaux dans le tissu urbain métropolitain. Mais en 1961, sous l'impulsion de la préfecture de la Seine, l'Institut national de la statistique et des études économiques (INSEE) et l'Institut d'aménagement et d'urbanisme de la région Île-de-France (IAURP, aujourd'hui Institut Paris Région) produisent pour 71 communes de la Seine ainsi que Paris ${ }^{4}$ des traitements statistiques du recensement de 1954 à l'échelle de l'îlot urbain, détaillant les catégories socioprofessionnelles mais aussi l'équipement et le confort des logements ainsi que des informations sur l'ancienneté des constructions (figure 1). L'échelle de l'îlot par sa précision ${ }^{5}$ permet d'appréhender la spatialisation des structures sociales. C'est en 1946 que l'îlot a été utilisé pour la première fois de manière systématique comme unité élémentaire de recensement pour la ville de $\mathrm{Pa}$ ris. En 1954, son emploi a été étendu à la majeure partie des communes suburbaines de la Seine. 


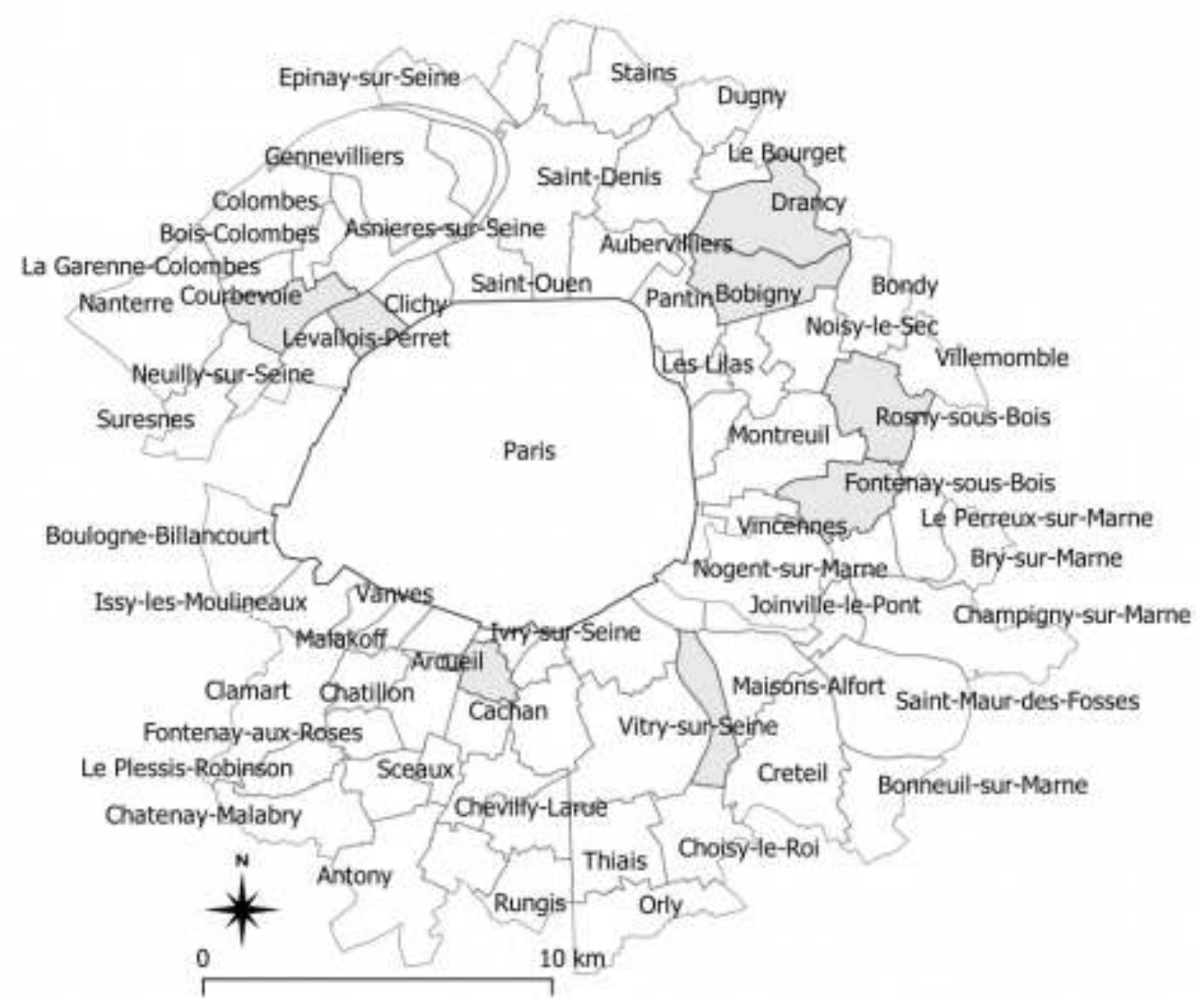

Sur les 80 communes du département de la Seine, 71 fascicules comprenant l'enquête ont été retrouvés. En gris, les communes absentes du recensement.

Carte des auteurs

Pour chaque commune, quatre tableaux traitent successivement de la population, de la population active, du logement et du bâti. Au total, une multitude de variables qui permettent d'aborder des aspects très divers dans une perspective spatiale fine. Le premier tableau concerne la population. Outre la répartition par sexe et par âge, le recensement indique, uniquement pour Paris, le nombre d'étrangers et, pour chaque îlot du département, celui des "Musulmans d'Algérie ». Le recensement différencie les types de ménages, ceux dits " ordinaires " selon l'INSEE et les ménages collectifs (hôtels, pensions, communautés religieuses, etc.). Le deuxième tableau, celui de la population active, donne la répartition des actifs selon les toutes nouvelles catégories socioprofessionnelles conçues par l'INSEE en 1954. Les individus sont classés selon leur situation professionnelle en tenant compte de plusieurs critères : le métier proprement dit, l'activité économique, la qualification, la position hiérarchique et le statut privé ou public (Desrosières et Thévenot 200o). Le troisième tableau renseigne le degré d'occupation des logements et le nombre de pièces. Il recense également leur équipement (eau, gaz, électricité, w.-c. particuliers, douche, chauffage central, téléphone). On peut ainsi savoir combien de logements disposaient de l'eau courante ou du chauffage central dans chaque îlot. On connaît le nombre de logements en situation de surpopulation admissible ou critique selon les critères de l'INSEE. Le dernier tableau concerne le bâti, en particulier, la nature (maisons d'habitation, hôtels, etc.), la date de construction ou le nombre d'étages des bâtiments. Ces 72 registres agrégeant près de 15 ooo îlots se font ainsi le reflet des préoccupations de l'administration au début des 
années 1960. Outre les traditionnelles mesures des classes d'âge et des catégories socioprofessionnelles, la question du logement, de la qualité et de la modernité du bâti y apparaît centrale.

L'exploitation des registres du recensement n'a donc pas nécessité une phase de construction de base de données et de réflexion particulièrement complexe, tant on a pu aisément calquer l'organisation des registres au sein d'un SIG. Néanmoins, elle a impliqué un travail important de saisie des données. Cette tâche avait déjà été réalisée pour l'ensemble de Paris dans le cadre de l'écriture de l'Atlas des Parisiens (Pinol et Garden 2009). La masse d'information restante à traiter (71 communes, 11 ooo îlots) interdit cependant toute retranscription manuelle et implique un travail en deux phases : la numérisation et l'océrisation ${ }^{6}$.

Les 71 fascicules concernant les villes de banlieue ont été minutieusement numérisés. À partir du logiciel ScanSnap ${ }^{7}$, nous avons ensuite procédé au traitement des images (redressage des pages, recadrage, unification de la lumière, métadonnées...) et produit 71 documents PDF qui pourront être reversés à la bibliothèque de l'Hôtel de Ville. L'étape essentielle du projet correspond au travail de reconnaissance des caractères, à partir du logiciel OCR Nuance, permettant de transformer le document PDF en tableur éditable. La principale difficulté à laquelle nous avons été confrontés réside justement dans les erreurs produites lors de cette phase d'océrisation. Malgré des résultats très satisfaisants, des coquilles apparaissent régulièrement : une confusion entre des 3 et des 5 ou des 8, et des ajouts de signes typographiques aux chiffres rendant impossible leur traitement quantitatif. D'autre part, l'absence de ligne horizontale dans les registres a souvent posé des difficultés de lecture pour le logiciel qui a parfois fusionné plusieurs lignes entre elles. Cette multitude de petites erreurs a nécessité un important travail de relecture. L'ensemble du travail d'océrisation et de relecture a pris autour de deux mois de travail à temps complet, dont près de $70 \%$ de ce temps consacré au minutieux travail de relecture.

La phase de développement de la base de données achevée, il fut possible de passer à la construction du SIG à proprement parler. Pour ce faire, il était nécessaire de vectoriser les 10850 îlots des communes de banlieue. L'idée de départ était de partir des îlots actuels, vectorisés par l'Atelier parisien d'urbanisme (Apur) en faisant le pari que les îlots d'aujourd'hui sont le résultat de divisions parcellaires de ces 50 dernières années, permettant ainsi, à partir d'outils de fusion, d'aisément s'y retrouver. La pratique fut tout autre. Certes, la majorité des îlots sont bien le résultat de ces phénomènes d'évolution du parcellaire, cependant de trop nombreux cas particuliers ont empêché une systématisation de la méthode. Et encore, c'est sans compter les immenses modifications du tissu urbain survenues lors de la création des axes autoroutiers, des lignes de RER ou de la construction de certains grands ensembles. Nous avons été contraints d'adopter une méthode classique de géoréférencement de l'ensemble des plans des communes annexés aux fascicules, puis d'un dessin vectoriel manuel pour tous les îlots. La difficulté de numériser ces larges feuillets accrochés au registre a produit de nombreuses imprécisions, tout comme l'assemblage de l'ensemble des communes entre elles. Ces difficultés ont nécessité de nombreux ajuste- 
ments manuels. Ce travail de vectorisation des îlots du département de la Seine a là encore nécessité deux mois de travail à temps complet (figure 2).

FIGURE 2. DU REGISTRE AU SIG

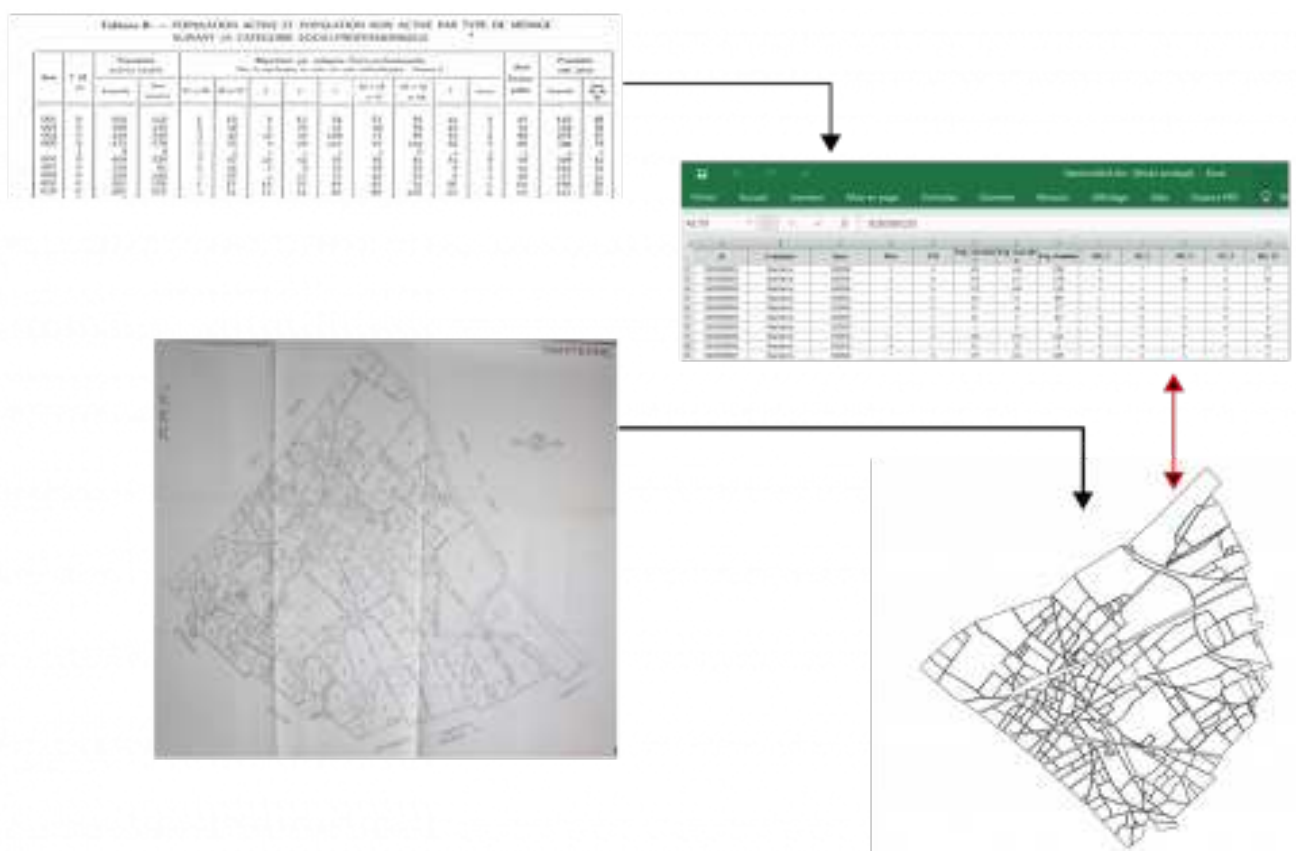

Ce schéma montre les deux étapes de traitement des données du recensement. D’abord l'océrisation des tableaux en base de données au format Excel, puis la vectorisation des îlots avec le logiciel QGIS. Les données et le plan vectoriel ont été connectés à partir d'un identifiant commun.

Schéma des auteurs 


\section{Les photographies aériennes et leur géoréférencement}

En parallèle de ce travail sur les données du recensement élaboré par le projet Seine 54, le programme ALEGORIA travaille à la valorisation des fonds iconographiques anciens. Le corpus est constitué de photographies aériennes représentant la France à différentes périodes, issues des fonds de l'IGN et l'AD92. Depuis les années 1920, l'IGN a régulièrement procédé à des relevés du territoire national via des vues aériennes verticales, mais aussi obliques. Ces dernières étaient alors centrées sur des zones spécifiques telles que celles subissant des changements importants (destructions, reconstructions...). Les vues verticales permettent de se positionner facilement et leur lecture est très similaire à celle d'une carte. Contrairement aux images obliques, elles offrent une représentation exhaustive du territoire. En revanche, elles permettent une meilleure perception de la topographie ou de l'architecture par exemple, car leur angle de vue est plus proche de celui de nos yeux, du fait d'une prise de vue à plus basse altitude (Humbert, Courtot et Renard 2013). Dans notre étude, nous nous sommes concentrés sur ces dernières, dont les caractéristiques se révèlent particulièrement intéressantes pour les analyses historiques et sociologiques. La collection étudiée se compose d'environ 30 ooo clichés numérisés, dont environ 700 pour Nanterre (voir quelques exemples dans la figure 3) et environ 200 qui concernent plus spécifiquement ses bidonvilles. D’autres collections existantes, hébergées par divers GLAM (Galleries, Libraries, Archives and Museums) pourraient également être envisagées pour élargir le cadre de cette étude.

FIGURE 3. EXEMPLES D'IMAGES AÉRIENNES OBLIQUES DE L'IGN
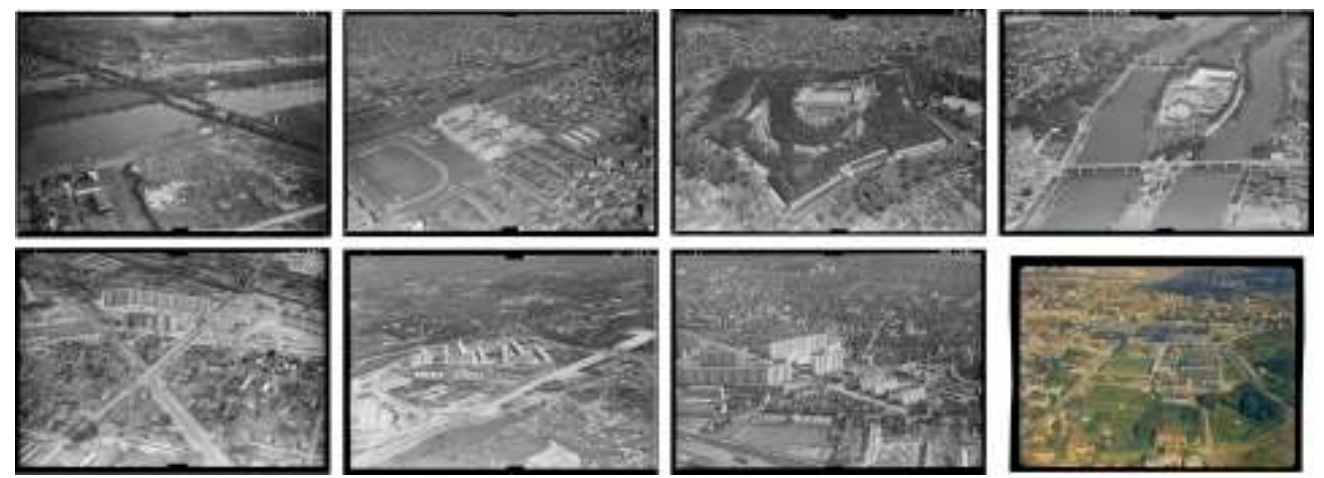

L'ensemble de ces vues montrent à voir différentes zones de Nanterre sur la période 19471960.

(C) IGN

Les travaux présentés ici supposent la géolocalisation dans l'environnement $3 \mathrm{D}$ de ces contenus photographiques. Différents niveaux de localisation sont généralement disponibles :

- Tout d'abord, un niveau de métadonnées textuelles (nom du département, de la ville...). Un outil de géocodage possède une base de référence qui permet de faire correspondre ces noms de lieux à des coordonnées $2 \mathrm{D}$, généralement placées au centre de l'emprise spatiale de ces lieux. C’est souvent le degré de localisation associé aux collections des GLAM. 
- Dans un deuxième niveau, une pose (une position 3D et trois angles d'orientation) déterminée au moment de l'acquisition est connue avec plus ou moins de précision. C'est le cas des collections acquises par les agences cartographiques comme l'IGN.

- Pour finir, certaines images peuvent être intégralement dénuées de toute information de localisation. Pour remédier à cela, différentes approches existent pour estimer une première pose pour l'image, chaque approche ayant différents degrés d'automatisation et d'interaction avec l'utilisateur, comme nous le verrons ci-après. Afin d'automatiser le processus de géolocalisation précise des images, un large spectre de techniques a été développé en photogrammétrie, vision par ordinateur et robotique ; plusieurs états de l'art existent sur le sujet : Lowry et al. (2016), Garcia-Fidalgo et Ortiz (2015), Brejcha et Cadik (2017), Piasco et al. (2018).

Une fois ces deux jeux de données géoréférencés, il était nécessaire de faciliter leur croisement pour permettre une analyse fine de ces phénomènes. La plateforme iTowns a permis leur appariement et a surtout offert un outil de visualisation immersif, aidant le chercheur à prendre conscience de l'espace.

\section{Un outil de covisualisation des données spatiales hétérogènes}

La plateforme iTowns a été mobilisée par les chercheurs en SHS des deux projets pour qu'ils exploitent leurs jeux de données, en les inscrivant dans l'espace. iTowns est effectivement une plateforme de visualisation Web interactive et immersive permettant à l'utilisateur d'afficher différentes images et de naviguer entre elles - qu'elles soient aériennes obliques, aériennes verticales ou même terrestres - ou couches de données statistiques, projetées au sein d'un même modèle $3 \mathrm{D}$ de référence représentant la Terre. Le retour d'expérience des usages des chercheurs en SHS a permis aux ingénieurs de l'IGN d'affiner la plateforme et de proposer de nouveaux outils de navigation adaptés aux pratiques de la recherche en sciences sociales. La visualisation de données, parfois hétérogènes, dans un cadre géoréférencé est donc un enjeu important, en particulier pour les humanités numériques. Un certain nombre de plateformes ont ainsi été développées ces dernières années pour répondre à ce besoin.

\section{État de l'art}

On peut distinguer deux types de plateformes : celles en deux dimensions permettant une représentation à plat de photos ou de plans et les premières expériences de plateformes 3D. 


\section{Plateformes 2D}

Une première catégorie d'applications se concentre sur les vues aériennes verticales telles que les orthomosaïques. Citons tout d'abord le projet Planet Explorer ${ }^{8}$ qui permet à l'utilisateur de visualiser des images satellites du monde entier, couplant cela à des outils permettant de les comparer. De manière similaire, de nombreuses agences nationales de cartographie valorisent leurs données anciennes via des portails Web (en particulier des cartes et des photographies aériennes). On peut donc y visualiser, comparer et même télécharger des données anciennes à l'instar du portail « Remonter le temps ${ }^{9}$ " de l'IGN où l'on peut retrouver des données $\mathrm{du} \mathrm{XVII}^{\mathrm{e}}$ siècle à nos jours. Ces plateformes ont pourtant quelques inconvénients : l'utilisateur ne peut pas visionner ses propres images, la visualisation n'est qu'en $2 \mathrm{D}$ et toute la donnée a été géolocalisée manuellement.

Une seconde catégorie se concentre sur la simple localisation de données de différents types (images, cartes, statistiques ou même articles de recherche). Les plateformes sont nombreuses et bien souvent sont au service d'une communauté en particulier. On peut ainsi citer Navigae ${ }^{10}$ qui permet aux utilisateurs de visualiser des images ou des cartes anciennes grossièrement géolocalisées au sein d'une application cartographique. Le groupe de recherche Inventer le Grand Paris ${ }^{11}$ a aussi développé un portail qui localise les projets de recherche concernant les métropoles mondiales. La plateforme devient donc un filtre spatiotemporel de ces projets.

Pour finir, d'autres plateformes se concentrent sur les photographies et leur visualisation dans un paradigme mixant $2 \mathrm{D}$ et $3 \mathrm{D}$. Tout d'abord, le projet Mapillary ${ }^{12}$ qui localise des clichés pris au niveau de la rue dans le monde entier et qui permet de les visionner de manière fluide en naviguant entre images de proche en proche. Navilium ${ }^{13}$, quant à elle, se veut une plateforme collaborative avec des documents visuels localisés datant $\mathrm{du} \mathrm{XIX}^{\mathrm{e}}$ siècle. Il y est même possible de comparer visuellement " côte à côte " en $2 \mathrm{D}$ la vue ancienne et celle de Google StreetView à la même position. Le projet HistoryPin ${ }^{14}$ permet aux utilisateurs de créer des collections d'images similaires, soit par leur localisation, soit par leur thématique. Elles sont également " épinglées " sur une carte $2 \mathrm{D}$, mais on peut aussi les afficher dans Google StreetView. L'estimation de la pose du cliché est rudimentaire, c'est une simple superposition de l'image sur une position de caméra StreetView. Dès lors, il est impossible de se mouvoir dans la scène $3 \mathrm{D}$, chaque déplacement de la caméra de l'utilisateur fait perdre de vue le document. Pour finir, on peut présenter le projet $A r$ chiMediaL ${ }^{15}$ qui cherche à interconnecter de manière automatique des images pour des études comparées du bâti ancien et contemporain. Pour cela, une plateforme d'annotation (construite en partie sur le visualiseur de Mapillary) a été développée et permet d'enrichir sémantiquement les images. Les photographies sont donc référencées comme prises depuis la rue ou prises en intérieur et les changements éventuels sont enregistrés sémantiquement (destruction partielle, nouvelle construction...). Ces images peuvent ensuite être utilisées en conjonction avec d'autres données historiques issues de domaines de recherche variés. Cependant, 
la plateforme ne permet pas d'afficher d'autres types de données anciennes et pour ces dernières il faut utiliser d'autres moyens de visualisation.

\section{Plateformes 3D}

Le nombre de plateformes en ligne utilisant un environnement 3D est bien plus réduit. L'utilisation de la 3D survient naturellement, en lien avec la nature de la donnée manipulée (par exemple, les objets architecturaux en trois dimensions). C'est le cas de la plateforme collaborative Aïoli ${ }^{16}$, par exemple, qui vise la documentation et le partage de notre patrimoine architectural. En alternant entre visualisation $2 \mathrm{D}$ et $3 \mathrm{D}$, l'utilisateur peut annoter les photographies et les modèles 3D, ces annotations se propageant ensuite à tous les utilisateurs, mais aussi de manière automatique à d'autres formes de documentations ou modèles d'un même objet d'étude. Par ailleurs, la navigation multitemporelle est possible, par exemple pour évaluer l'évolution du processus de conservation et identifier de potentielles dégradations.

Un autre visualiseur 3D est UrbanHistory4D, dédié à la présentation du patrimoine urbain de Dresde en Allemagne. Différents documents, peintures, esquisses, etc., datées de 1675 pour les plus anciennes, issues de différentes collections iconographiques sont positionnées précisément et visualisées dans le modèle $3 \mathrm{D}$ d'un quartier de la ville. On peut visionner les diverses vues dans le contexte du modèle $3 \mathrm{D}$ des bâtiments. D'autres modes de visualisation sont possibles tels que la carte de chaleur de la distribution spatiale ou bien une " carte radar » des orientations des documents. Cependant, l'utilisateur ne peut pas afficher ses propres images pour les localiser et seule une petite zone d'étude sur Dresde est disponible.

Pour finir, Smapshot ${ }^{17}$ est une plateforme Web suisse qui fait participer l'utilisateur à la géolocalisation de représentations anciennes de paysages fournies par la bibliothèque de l'École polytechnique fédérale de Zürich ou l'office fédéral de topographie. L'utilisateur doit sélectionner au moins six correspondances entre l'image et le modèle 3D et également fournir la position approchée de la caméra prenant la photographie sur une carte en $2 \mathrm{D}$ et la direction approximative de prise de vue. La plateforme se concentre en revanche uniquement sur la Suisse et une équipe doit valider la localisation de l'image faite par l'utilisateur.

Le nombre grandissant de plateformes de visualisation témoigne de l'engouement pour ces outils. L'IGN a développé de son côté sa solution propre visant à créer un outil souple aux usages multiples correspondant aux missions de l'IGN. En particulier, l'accent a été mis sur le caractère immersif de l'expérience. 


\section{Développement d'une application Web de visualisation immersive}

Les différents outils formant cette plateforme reposent sur iTowns, une librairie open source (Devaux, Brédif et Paparoditis 2012) développée depuis 2008. Son utilisation à grande échelle est par ailleurs assurée par le fait qu'elle est le moteur de visualisation 3D du Géoportail de l'IGN. Faisant appel à des services Web géographiques, iTowns permet de représenter la Terre sous la forme d'un globe virtuel avec différentes données hétérogènes, comme de l'imagerie du sol (images, nuage de points 3D), mais aussi des données vecteurs (bâtiments, réseaux routiers, etc.), et cela à grande échelle avec une grande précision. Par ailleurs, cette librairie permet de naviguer de façon continue entre des points de vue au niveau de la rue et des points de vue aériens verticaux ou obliques, ce qui en fait un cadre de référence idéal pour nos outils.

Au sein de ce cadre de référence, différents " fonds de carte " sont disponibles pour s'adapter à la visualisation souhaitée avec, par exemple, des cartes anciennes ou plus récentes, des photographies aériennes verticales, mais également des ortho-images établies à différentes dates, permettant la comparaison visuelle du territoire à travers les époques. De la donnée vecteur peut également être projetée sur le modèle $3 \mathrm{D}$, ce qui apporte de nouvelles informations sur la zone d'étude, que ce soit simplement ses limites administratives ou bien des statistiques plus complexes, telles que celles issues du SIG de Seine 54 (figure 4). Tous ces types de données peuvent donc être covisualisés de manière fluide et immersive.

FIGURE 4. COVISUALISATION DE DONNÉES HÉTÉROGÈNES

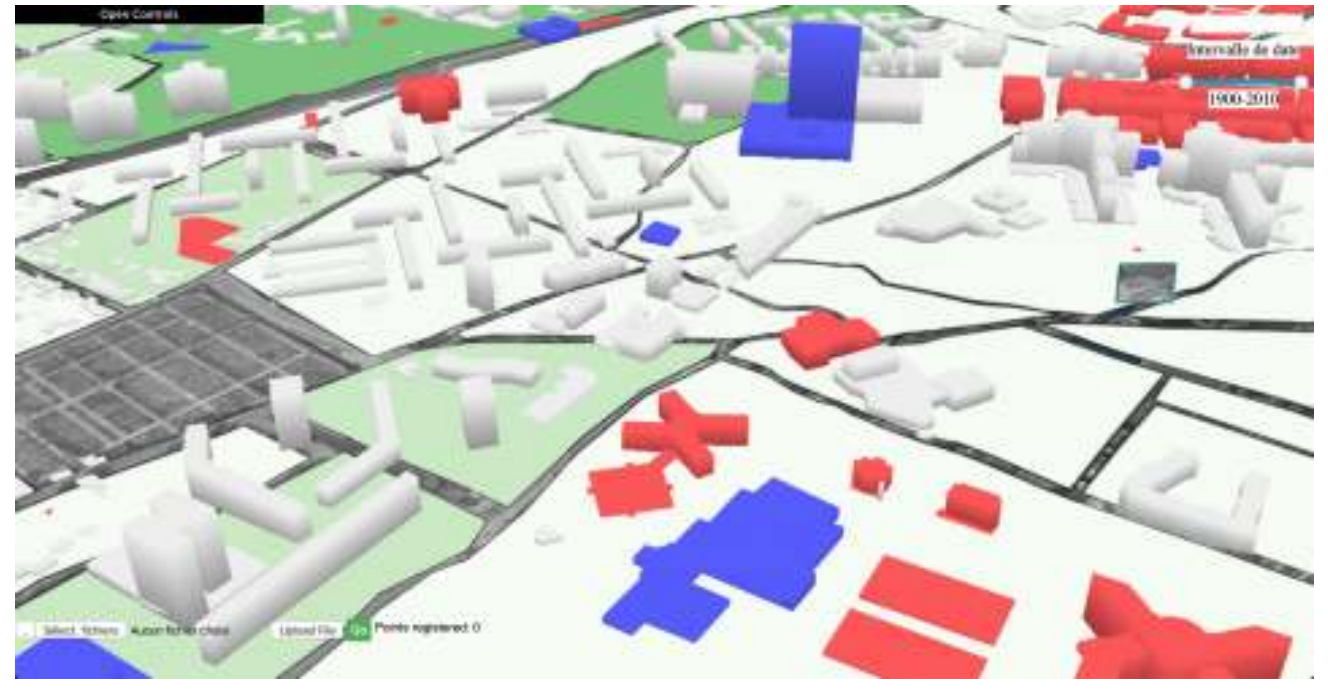

Cette figure présente une juxtaposition de données hétérogènes : en 3D, le bâti actuel, les îlots en dégradé de vert correspondent à un traitement statistique du recensement de 1954 et l'on voit apparaître à gauche une photographie aérienne. 


\section{La géolocalisation interactive des images dans la plateforme Web}

Dans le cadre de cette étude sur Nanterre, l'outil iTowns a permis de facilement replacer dans l'espace les photographies aériennes mobilisées. Effectivement, nombre des vues utilisées n'étaient pas précisément géolocalisées et orientées et elles étaient encore moins visualisables dans l'environnement 3D. Une fonctionnalité importante d'iTowns repose sur la possibilité pour l'utilisateur de géolocaliser lui-même les images anciennes. On s'appuie ici sur une méthode semi-automatique de géolocalisation, en particulier celle proposée dans Harrach, Devaux et Brédif (2019). De manière interactive, l'utilisateur sélectionne au moins quatre paires de points correspondants en $2 \mathrm{D}$ sur la photographie à localiser et en $3 \mathrm{D}$ sur le modèle $3 \mathrm{D}$ de référence (figure 5 ). Le modèle $3 \mathrm{D}$ exploité est le modèle numérique de terrain (MNT) utilisé pour créer le globe virtuel. Il est possible d'y ajouter d'autres informations, comme un modèle numérique de surface $\left(\mathrm{MNS}^{18}\right.$ ) ou des nuages de points $3 \mathrm{D}$ acquis par scan laser ou par une technique de vision par ordinateur (Structure from Motion). Dans notre cas, une représentation 3D des bâtiments a été exploitée dans l'objectif d'améliorer la précision de la géolocalisation. Les paires de points en correspondance sont ensuite utilisées en entrée d'un logiciel de photogrammétrie qui estime la pose du cliché. Le processus employé est la résection spatiale au sein de scènes architecturales (Busayarat 2006). Ce processus utilise la position des objets dans l'espace et la position de ceux-ci dans l'image pour déterminer les paramètres de la pose de la caméra (ou de l'image), à savoir trois positions et trois rotations.

FIGURE 5. LOCALISATION SEMI-AUTOMATIQUE D'UNE IMAGE DANS ITOWNS

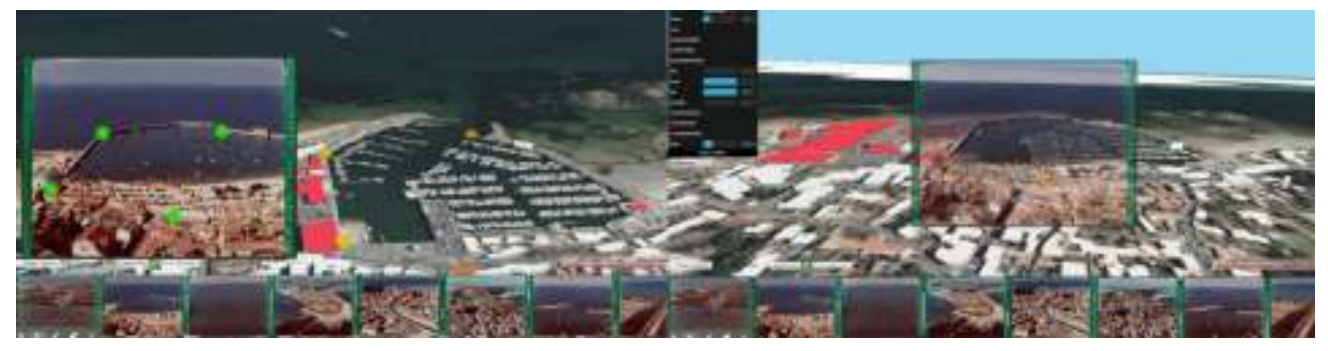

Ces deux exemples concernant le port de Noirmoutier représentent un exemple de la capacité de localisation des vues aériennes dans iTowns. À gauche, la sélection des paires de points $2 \mathrm{D}$ (vert) et 3D (orange) et, à droite, l’image géoréférencée : la caméra de visualisation est placée aux mêmes position et orientation que la pose estimée de l'image géoréférencée, de sorte à superposer le format en $2 \mathrm{D}$ et le monde virtuel 3D. Cette fonctionnalité permet de spatialiser aisément et rapidement d'importants corpus comme ceux mobilisés dans cette étude.

(C) IGN 


\section{Une navigation immersive à travers le temps et l'espace}

L'enjeu du projet était de covisualiser un très grand nombre d'images aériennes, associées à des données statistiques géolocalisées et datées. Pour faciliter le croisement de ces sources, il convient d'être capable de naviguer à travers le temps et l'espace de manière ordonnée, intuitive et ergonomique. iTowns offre de nombreuses fonctionnalités permettant cette navigation, mais l'observation de nos besoins et de nos pratiques a aussi permis de faire évoluer iTowns avec le développement de nouvelles fonctionnalités.

Lors de l'élaboration du projet, nous nous sommes en particulier appuyés sur un outil de visualisation de la plateforme qui permet une représentation globale de la donnée disponible. Ainsi, une carte de chaleur de la répartition des clichés sur l'ensemble du territoire (figure 6) a pu identifier les lieux riches en données et attirer l'attention sur certaines zones propices à l'étude à l'aide de l'outil de visualisation.

FIGURE 6. VISUALISATION DE LA DISTRIBUTION SPATIALE DES IMAGES À L'AIDE D'UNE CARTE DE CHALEUR

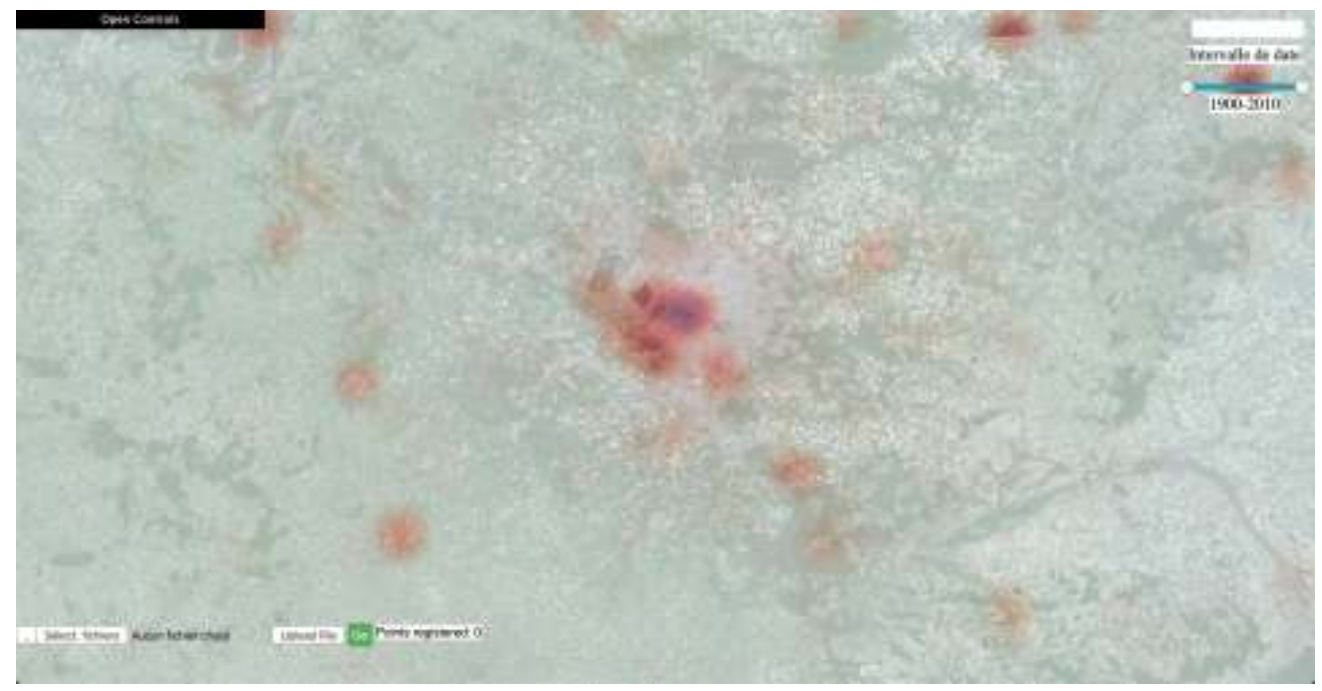

Carte de chaleur de la densité d'images disponibles. Leur forte présence pour l'Ouest parisien et, en particulier, Nanterre, nous a conduits à orienter nos recherches vers ce secteur de la métropole parisienne.

(C) IGN

Pour permettre la navigation la plus fluide possible, la plateforme procède à un filtrage des données pour traiter et télécharger uniquement les images du secteur sélectionné par l'utilisateur. À noter que les images ne sont pas téléchargées localement, mais seulement récupérées ponctuellement auprès de l'institution de conservation au moment de la visualisation, de manière à respecter les droits d'utilisation et de diffusion sur ces contenus.

Outre cette sélection spatiale, le travail réalisé sur les bidonvilles de Nanterre a fait émerger l'importance de disposer d'un outil de sélection temporel. Afin de ne visualiser que les données correspondant à la période souhaitée lors de l'étude, un curseur temporel permettant de sélectionner l'intervalle de temps de l'étude a été développé. Tout type de donnée qui possède une date sera ainsi affecté par ce choix. Cette fonc- 
tionnalité apparaît nécessaire lorsque l'outil est utilisé pour des études au spectre temporel assez restreint, comme l'analyse de la formation d'un bidonville. Cela permet en effet de trier rapidement les données intéressantes, mais aussi d'améliorer la visualisation en enlevant le "bruit » lié à la présence de données " parasites ». Ainsi, lors de l'étude de Nanterre, alors que de nombreuses représentations de l'IGN étaient disponibles pour Nanterre, pouvoir jouer avec l'intervalle temporel autour de 1954 permettait de se concentrer sur l'avant, le pendant ou l'après du phénomène étudié et de ne visualiser que la donnée pertinente pour notre réflexion.

Afin d'assurer une certaine fluidité dans la visualisation, différents points de vue (figure 7) s'offrent à l'utilisateur et les manières d'en changer s'avèrent également multiples. Il peut ainsi se mouvoir librement dans l'espace, visionnant les images à la position de leur acquisition, au milieu des autres types de données visibles. L'utilisateur peut ensuite cliquer sur une photographie pour se placer à la position de la caméra et ainsi visualiser la scène comme elle était à l'époque de la prise de vue. Cet outil simple à prendre en main a permis de sélectionner et d'étudier les images utiles à cette étude. Cette liberté de mouvement permettait de " tourner " autour du bidonville pour visualiser au mieux l'évolution et les déplacements de cette entité en constante mutation.

En outre, la fonction de transparence des couches de la plateforme facilite l'analyse de différentes images ou de différents types de données, ainsi que leur comparaison. Elle permet aussi une comparaison avec le bâti actuel, modélisé en 3D, donnant à voir la fulgurante mutation urbaine qui a pris place sur ce secteur ces cinquante dernières années. Enfin, un outil de géocodage a été développé afin de se rendre rapidement dans une autre zone d'étude en tapant son nom dans un champ de saisie.

FIGURE 7. DIFFÉRENTS POINTS DE VUE DE VISUALISATION
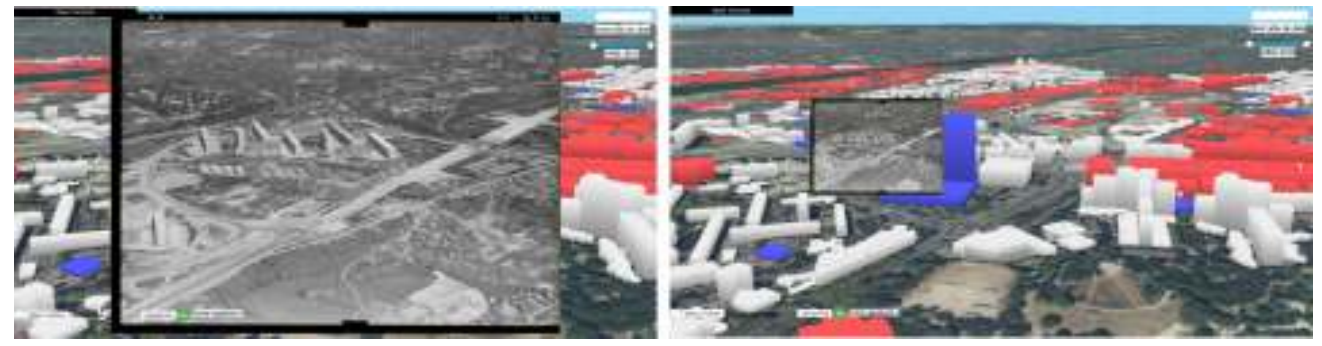

Exemple d'une navigation sur la plateforme iTowns. À gauche, on voit une photographie de la cité des Provinces françaises, mitoyenne du bidonville, depuis le point de vue de la caméra qui a pris la photo et, à droite, le point de vue libre permet de replacer cette photographie dans son contexte urbain actuel.

(c) IGN

Parallèlement, pour naviguer facilement dans la collection de photographies, un carrousel en bas de l'écran présente les images se trouvant dans la scène visualisée (figure 8). On peut ainsi les faire défiler et cliquer sur celle de notre choix. Si sa pose est connue, on se placera au niveau de l'image, comme expliqué précédemment. Sinon, l'interface de géolocalisation manuelle sera affichée afin de déterminer la pose si on le souhaite. Cela permet d'avoir un aperçu exhaustif du corpus mobilisé dans le projet. Par ailleurs, lors d'études sur des lieux très spécifiques comme dans notre cas, un autre outil peut être utilisé. Il sélectionne un point 3D dans le modèle et n'affiche que les images qui «voient " ce 
point. Cela met également à jour le carrousel, afin de naviguer entre les photographies pertinentes. Combiné avec la sélection temporelle, cet outil trie très rapidement les images pour trouver celles qui nous intéressent.

FIGURE 8. NAVIGATION ENTRE LES VUES ET CARROUSEL D'IMAGES

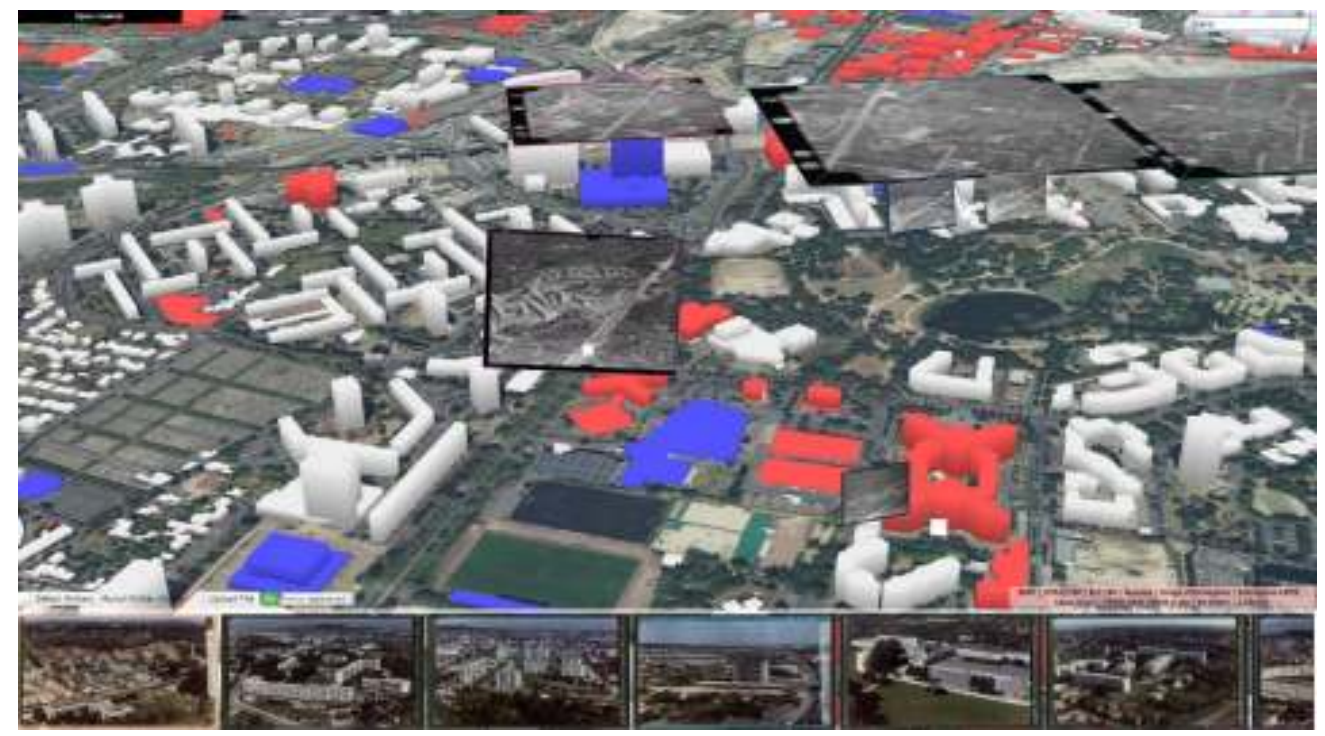

Le carrousel d'images permet de voir l'ensemble des photographies aériennes concernant un espace et une période donnés.

(c) IGN

iTowns se prête donc particulièrement à une analyse spatiale de jeux de données différents. Pour les chercheurs en sciences sociales, il peut effectivement représenter un instrument extrêmement puissant, autorisant une navigation entre des sources extrêmement diverses. Il permet d'aller plus bien loin que d'autres plateformes, telle que "Remonter le temps ", en offrant des possibilités de géoréférencement semi-automatisé et un cadre de visualisation immersif en $3 \mathrm{D}$.

\section{À la Folie, genèse d'un bidonville nanterrien}

À partir de l'outil iTowns, qui permettait la covisualisation des traitements statistiques réalisés sur le système d'information géographique exploitant les données issues du recensement de 1954 et des photographies aériennes géoréférencées et datées, nous nous sommes attachés à reconstituer le moment de cristallisation du bidonville de la Folie à Nanterre au milieu des années 1950. Notre objectif était de mieux comprendre la genèse et les raisons de la formation de cette forme urbaine si particulière. Par définition, la construction de ces habitats de fortune laisse peu de traces dans les archives et il est très difficile de documenter le moment de leur formation. Le croisement des recensements et des vues aériennes offrait une opportunité pour appréhender ces phénomènes avec un regard nouveau. 


\section{Nanterre en 1954}

Nanterre connaît d'immenses transformations au début du $\mathrm{xx}^{\mathrm{e}}$ siècle, suite à l'expansion urbaine et à l'industrialisation de l'agglomération parisienne. Au moment du recensement de 1954, Nanterre comptait 53 o11 habitants, soit presque quatre fois plus que la population de la commune en 1900 (14 140 habitants). À la date du recensement, Nanterre se trouve à la veille de changements majeurs puisque, dans les années 1950 et 1960, la ville devient un lieu clé de l'agglomération parisienne avec l'installation de l'université annexe à la Sorbonne et sa désignation comme chef-lieu du nouveau département des Hauts-deSeine qui s'accompagne de la construction d'une nouvelle préfecture. En outre, à partir de 1951, sous l'impulsion de Raymond Barbet, maire communiste, des milliers de logements sociaux sont construits. Toutes ces transformations ont lieu à l'écart du vieux village au nord de la commune et constituent un pôle incarnant la modernité urbaine de l'époque. Mais, dans le même temps, des dizaines de quartiers d'habitat informel et de bidonvilles se développent, abritant des Algériens récemment arrivés dans la métropole.

Le territoire de Nanterre en 1954 est typique des communes de banlieue parisienne. En grande partie, l'extension urbaine de la commune s'est appuyée sur le développement de vastes opérations de lotissements avec la construction de nombreux logements individuels de mauvaise qualité autour du centre-ville et de sa gare (Fourcaut 2000). Ces nouvelles formes urbaines se sont développées de manière concentrique autour du vieux centre villageois, au plus près de la gare. En marge de ces territoires, de nombreux terrains se trouvent dans une forme d'entredeux urbain. Ce sont encore des espaces essentiellement ruraux en déclin, où se mêlent terres agricoles et terrains vagues, avec parfois l'irruption d'un certain nombre d'industries qui s'installent sur ces îlots, en particulier ceux situés en bord de Seine. Les quelques logements sont différents de ceux du centre-ville. Ils apparaissent proportionnellement plus surpeuplés avec la présence de nombreux garnis. On perçoit ainsi dans les données du recensement une véritable fracture entre le Nanterre centre et le nouveau Nanterre environnant. 


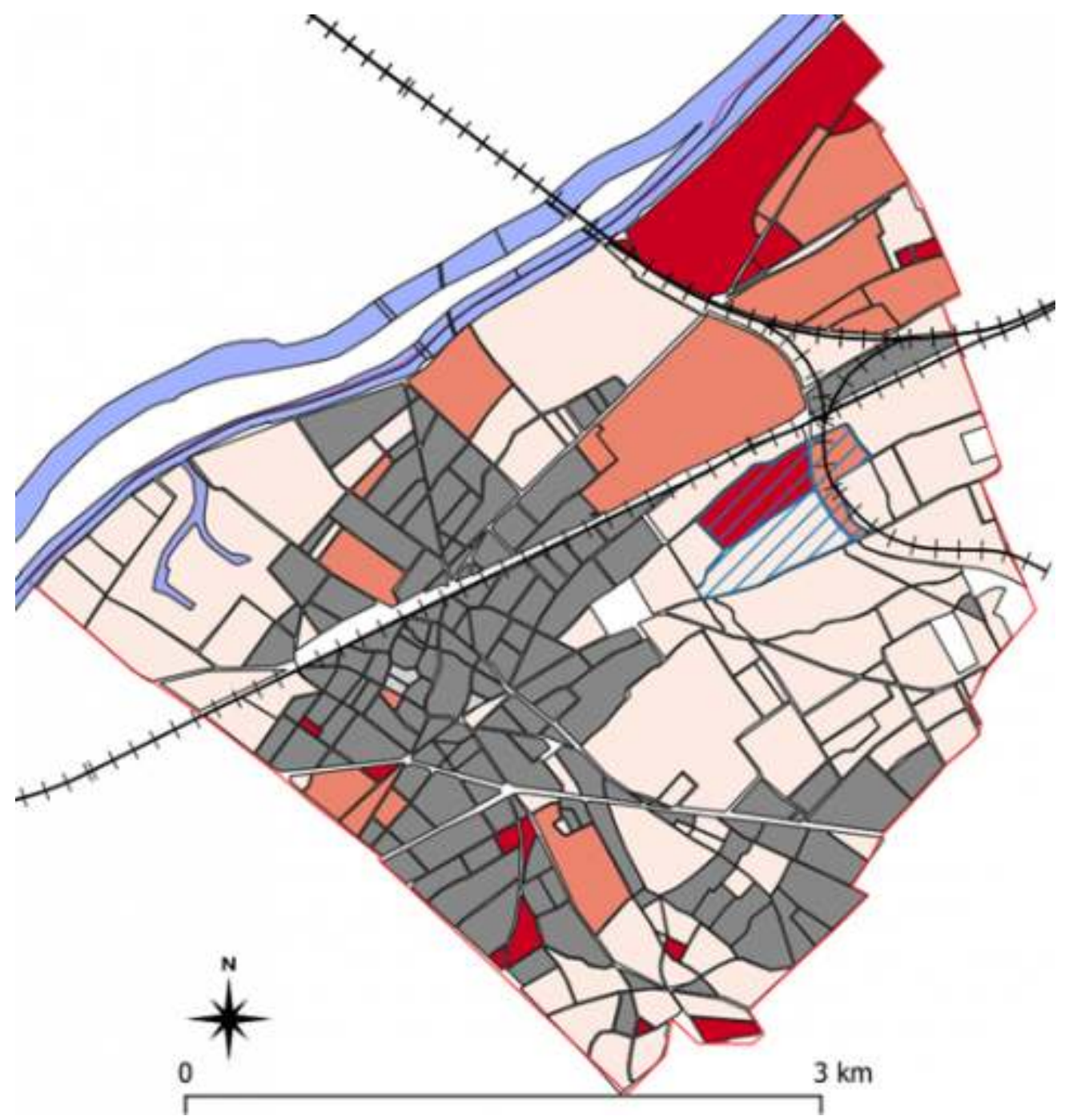

La carte met en évidence des groupes d'îlots (d'une même couleur) semblables au regard des variables considérées. Plus la couleur varie sur une échelle allant du gris au rouge, plus les îlots sont dissemblables. On voit ainsi des territoires en rouge et orange essentiellement au nord-est de la commune qui se différencient des îlots en gris au sud. Les îlots hachurés en bleu représentent la zone d'extension maximale du bidonville de la Folie dans les années 1960.

Carte des auteurs

Sur la figure 9, les îlots rouges incarnent le nouveau Nanterre en voie de transformation à la fois industrielle et urbaine, avec un habitat et une population plus fragile. Cette précarité se perçoit par la surreprésentation des hôtels garnis sur ces îlots, mais aussi par une proportion plus forte d'ouvriers non qualifiés. Les îlots rouges du centre sont souvent de petits îlots où la population se concentre au sein d'un ou deux hôtels garnis.

L'îlot 165, de couleur rouge et hachuré en bleu sur la carte, correspond approximativement au site de la cité des Provinces françaises. C'est au sud de cette cité que les premières cabanes de la Folie ont vu le jour. Dans l'îlot 165, on compte alors 240 individus pour à peine 35 logements. Un petit tiers de ces logements se trouve en situation de surpopulation critique ${ }^{19}$, pour seulement un cinquième en moyenne dans l'ensemble de la commune. Ce sont des hommes à plus de $70 \%$ et un quart d'entre eux 
ont entre 20 et 24 ans. Enfin, les Musulmans d'Algérie sont beaucoup plus nombreux qu'ailleurs, ils représentent $48 \%$ de la population pour $4 \%$ en moyenne à Nanterre.

\section{Les Algériens à Nanterre}

Pendant vingt ans, dans les années 1950-1960, la France favorise la venue de travailleurs étrangers pour les besoins de l'industrie en pleine croissance. Les ressortissants nord-africains, issus des anciennes colonies françaises, fournissent une main-d'œuvre abondante, peu qualifiée et bon marché pour les secteurs du bâtiment et de l'automobile. Les immigrés algériens, qui peuvent circuler librement jusqu'en 1962, fin de la guerre d'Algérie, continuent d'affluer en nombre croissant. Ils sont 210 ooo en 1954, 460 ooo en 1964, puis plus de 700 ooo en 1975. Cette immigration maghrébine des années 1960 demeure alors majoritairement le fait d'hommes seuls ${ }^{20}$. En 1964, 43 \% des Algériens de France vivent dans des bidonvilles, ceux de Nanterre abritent 14 ooo personnes.

Le problème des bidonvilles qui surgit à la fin des années 1950 est intrinsèquement lié à la question de la présence des Algériens en France (Blanc-Chaléard 2016). Même si l'habitat précaire et fragile est une constante de l'agglomération parisienne du XIX $^{\mathrm{e}}$ siècle et du début du $\mathrm{xx}^{\mathrm{e}}$ siècle et n'intéresse que peu l'État. Mais les Algériens dans les années 1950 sont une population considérée comme particulièrement inquiétante par les pouvoirs publics, à mesure de l'aggravation des troubles en Algérie. Leur concentration au sein de baraquements préoccupe l'État. Le terme même de bidonville est une importation en métropole d'un concept colonial développé dans les années 1930 (Barros 2012). L'administration multiplie alors les enquêtes et s'empare du problème des bidonvilles. Les traitements statistiques de l'INSEE et de l'IAURP s’inscrivent dans cette démarche (Barros et Cohen 2019). Pour les pouvoirs publics, il s'agit de dénombrer les Algériens et de spatialiser leur présence dans l'espace urbain. Pour les nommer, ils reprennent la catégorie coloniale spécifique de Français que sont les "Musulmans d'Algérie ». Français de jure, leur nationalité est vidée de facto de sa substance (Escafré-Dublet, Kesztenbaum et Simon 2018 ; Weil 2005 ; Barros 2005). 


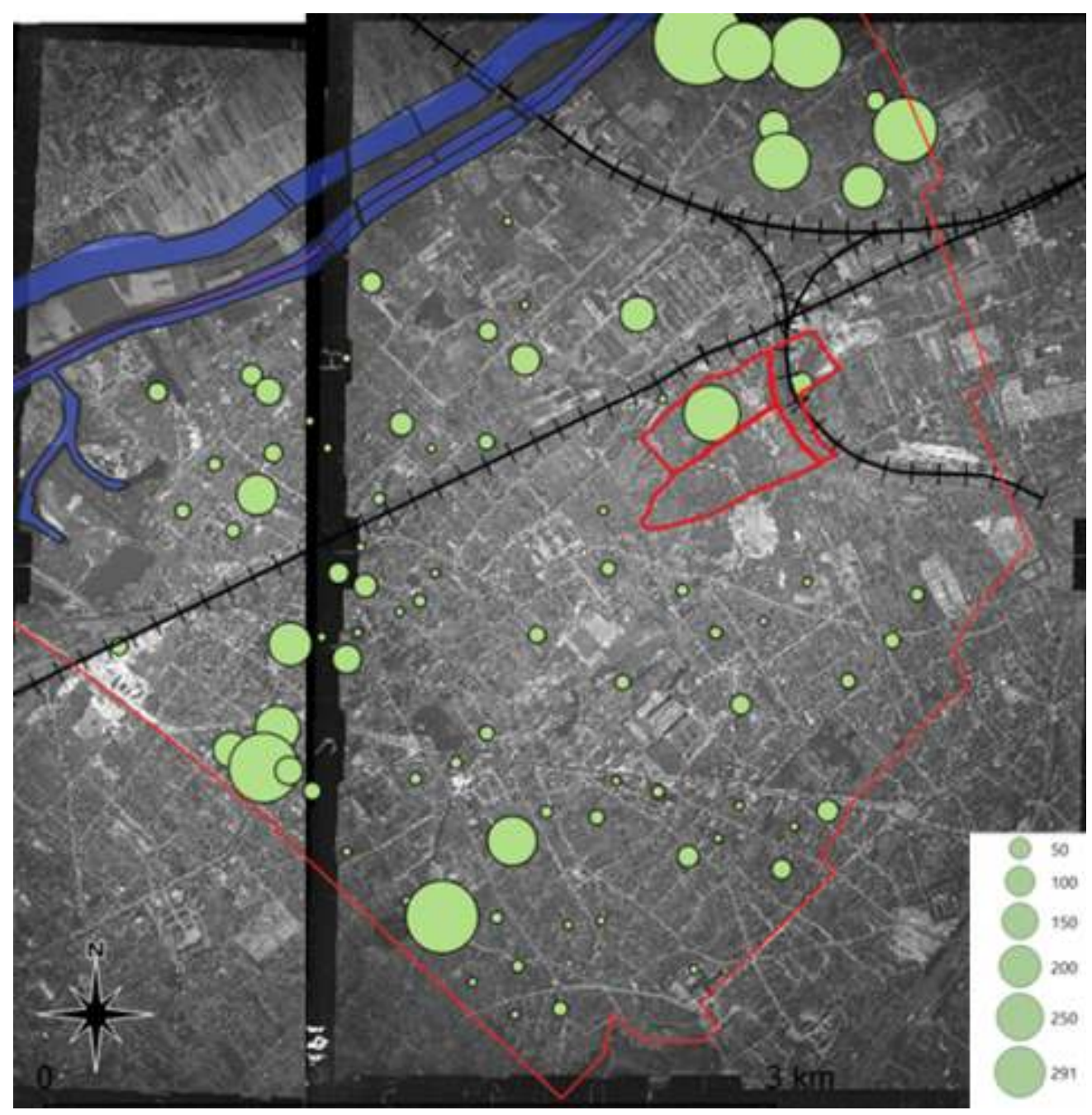

Cette carte représente le nombre d'Algériens recensés sur le territoire de Nanterre en 1954. On constate une concentration dans le centre, mais surtout dans le nord de la commune, pourtant relativement peu bâti. Sur le territoire du futur bidonville de la Folie, deux garnis abritent 115 d'entre eux.

Carte des auteurs

Le recensement des Algériens est peu précis tant l'opération de dénombrement fut compliquée par les conditions de logement, le mode de vie des Algériens marqué par les $3 \times 8$ et leurs réticences face à une opération perçue comme une forme de contrôle social. L'INSEE évalue que cette population est sous-estimée de 20 à $30 \%$ (Barros et Cohen 2019). La cartographie des Algériens recensés (figure 10) montre qu'ils semblent se concentrer au nord-est de la ville, au sud-est et à proximité de l'actuelle gare de RER. À l'aide des photographies aériennes de 1954, on observe que ces quartiers sont encore peu urbanisés, on y trouve essentiellement des terres agricoles et parfois de grands bâtiments, des hangars ou des sites industriels. Si l'on considère en particulier les îlots 164 et 165 (encadrés en rouge), où les premières cabanes du bidonville de la Folie commencent à apparaître, les Algériens y sont proportionnellement plus présents que sur le reste du territoire de la commune. Si seuls 14 immeubles sont recensés abritant 35 logements, il semblerait que les 115 Algériens soient logés dans les deux immeubles présentés comme des hôtels garnis ${ }^{21}$ (figure 11) attestant ainsi l'idée 
qu'ils sont surpeuplés. Les bidonvilles se seraient constitués d'abord dans les arrière-cours de ces immeubles avant d'investir les terrains vierges environnants (Sayad 1995).

FIGURE 11. CARTE STATISTIQUE DU NOMBRE D'HÔTELS GARNIS PAR ÎLOT

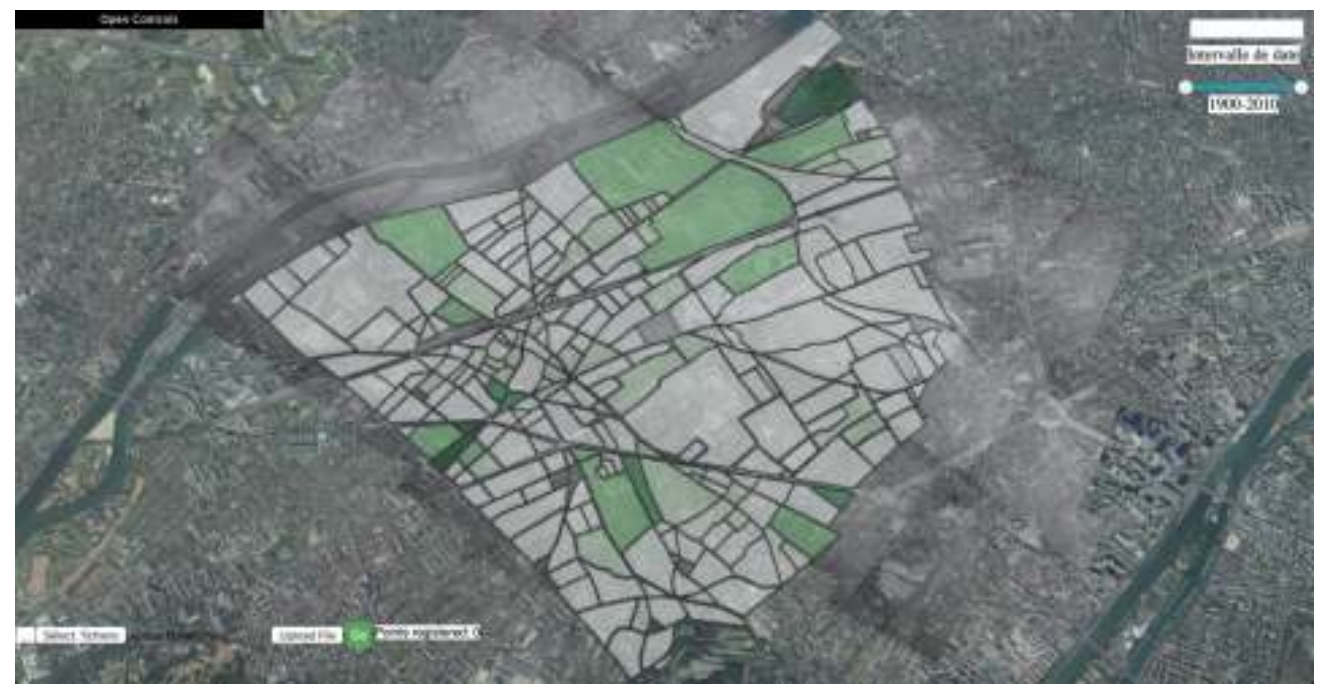

Depuis la plateforme iTowns, il est possible d'insérer des cartes statistiques réalisées à partir du SIG. À partir d'un outil de transparence, on peut ainsi jouer entre les différentes données photographiques et statistiques.

(c) IGN

\section{La formation du bidonville}

L'usage des photographies aériennes complète ces travaux ainsi que les résultats statistiques du recensement. À partir de l'outil iTowns, nous avons retracé la chronologie de la constitution du bidonville de la Folie. La plateforme a aussi permis de placer ce corpus photographique dans une dimension diachronique. Un curseur temporel, ajouté aux outils du carrousel ou de la représentation conique, offre la possibilité d'une navigation facilitée dans le temps et l'espace (figures 7 et 8). Pour les historiens, cela représente une façon d'appréhender ces fonds photographiques selon des modalités nouvelles et particulièrement heuristiques.

Le premier survol en 1954 donne à voir un terrain parsemé de rares constructions, correspondant à la description du bidonville par Laurent Maffre :

La Folie était un îlot perdu au milieu de nulle part. Autour, c'était un immense terrain vague [...] on parle d'un temps qui n'est pas si éloigné, où autour de Paris, c'était encore des petits pavillons, des petites cultures et un espace qui allait être terrassé et radicalement transformé en 30 ans. On sent que c'est un lieu à l'écart. Il y a une certaine modernité des bâtiments qui s'affiche en arrière-plan, avec quelques grues. Mais on sent qu'on est un peu perdu. On est dans un entre-deux, un espace d'attente et de transition entre l'Algérie et la vie future. (Maffre 2012)

Cependant, les grandes opérations urbaines du nord de Nanterre débutent. On voit ainsi sortir de terre le boulevard des Provinces françaises, première pièce d'une grande opération urbaine consistant à l'ouverture d'un nouvel axe structurant pour Nanterre avec l'avenue Jo- 
liot-Curie et la construction d'une vaste cité de logements sociaux (figure 12). Les travaux ne font que commencer et pourtant, à proximité d'une petite maison, peut-être un hôtel garni occupé par des travailleurs algériens, les premières cabanes de fortune apparaissent.

FIGURE 12. CABANONS DE FORTUNE EN 1954 SUR LE SITE DE CONSTRUCTION DE LA CITÉ DES PROVINCES FRANÇAISES

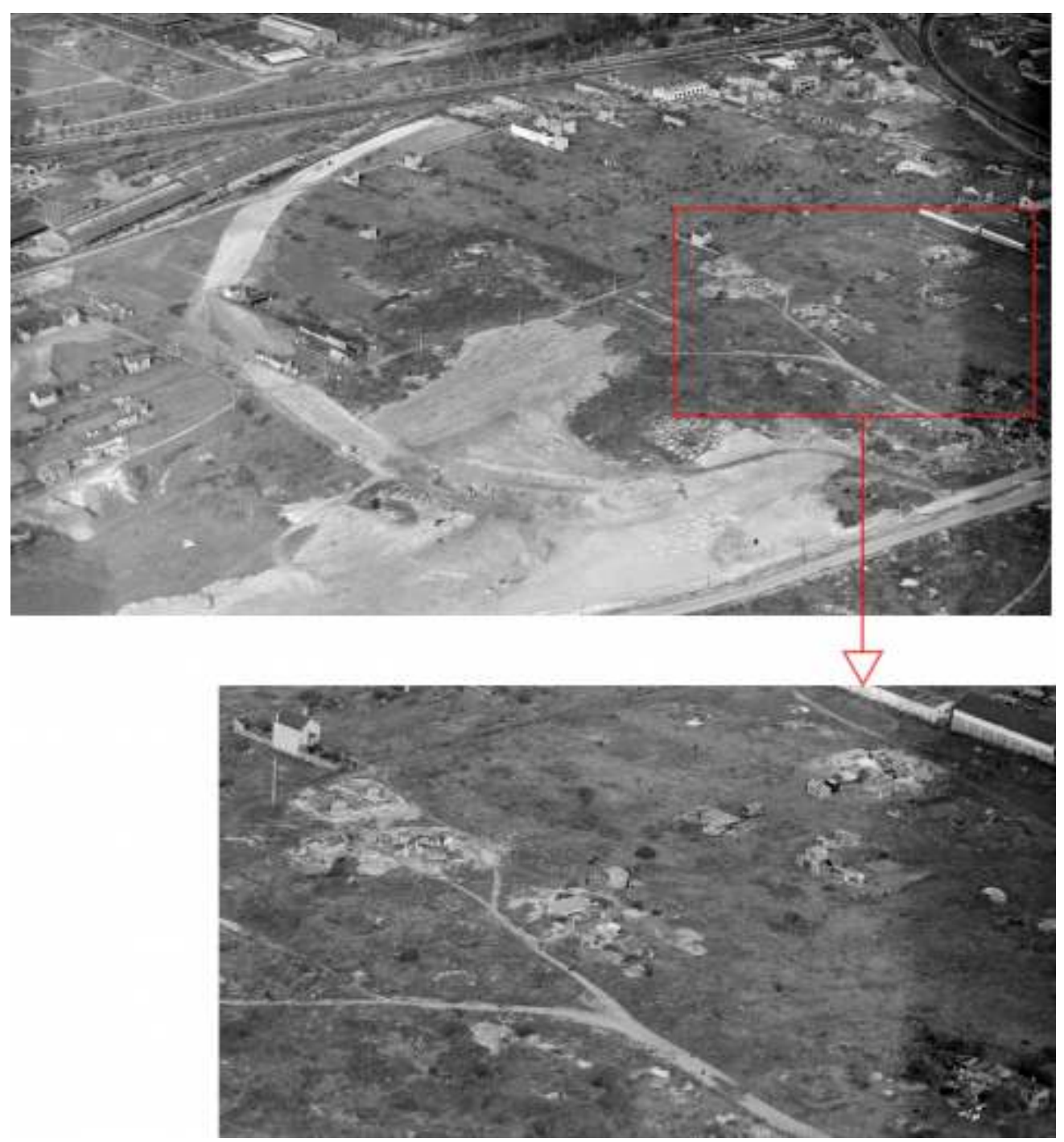

À partir des recherches sur l'outil iTowns, les premières traces du bidonville de la Folie ont été retrouvées au moment même des premiers travaux sur la zone.

(c) IGN

Un an plus tard, les fondations de la cité de logements débutent. La précédente maison a déjà disparu, certainement détruite pour faire place nette aux nouvelles constructions (figure 13). Les cabanes pourtant demeurent et s'agrègent les unes aux autres pour former des ensembles de plus en plus cohérents. 


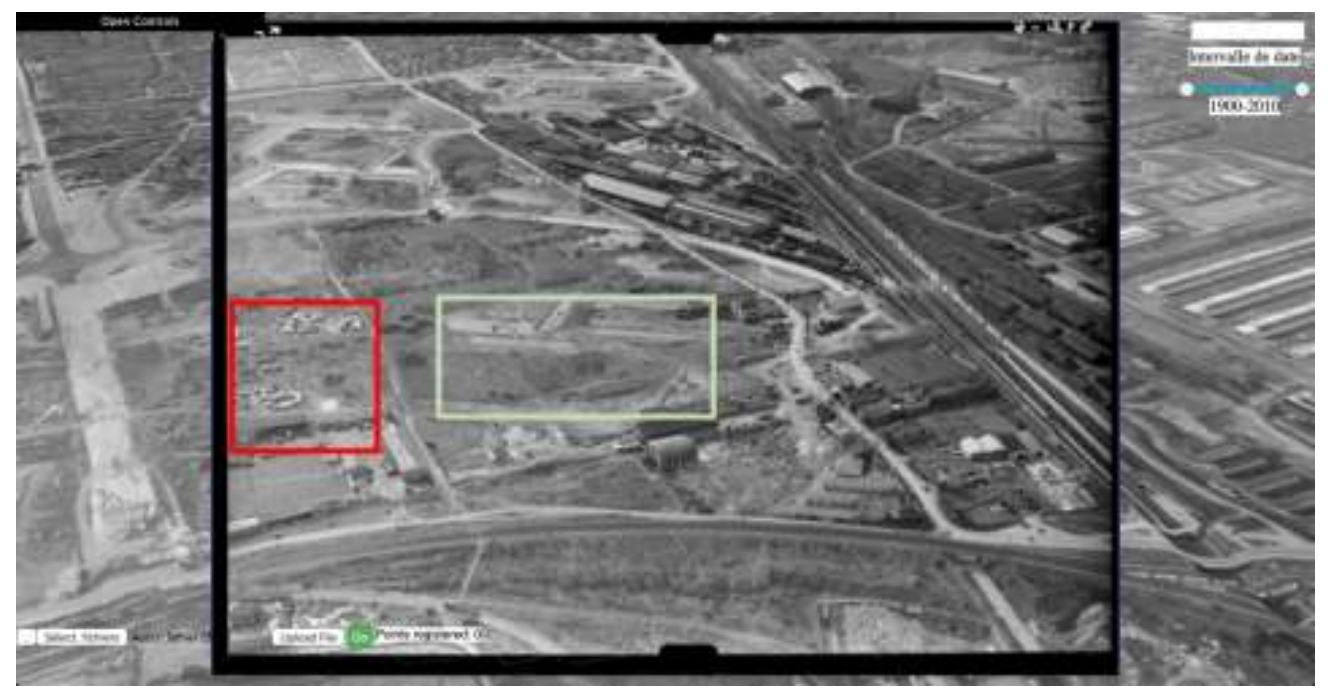

Vue d'une photographie aérienne oblique géoréférencée depuis la plateforme iTowns. En rouge les premières baraques, en vert, les fondations des Provinces françaises.

(C) IGN

En février 1956, avec l'accélération des chantiers, les besoins en maind'œuvre redoublent. Les cabanes prolifèrent et constituent un ensemble qui s'étend de plus en plus et débordent de l'autre côté de l'avenue JoliotCurie (figure 14). En quelques mois, ce sont des dizaines de nouvelles constructions informelles qui se créent.

FIGURE 14. Le CHANTIER DE LA CITÉ DE PROVINCES FRANÇAISES LE 1ER FÉVRIER 1956

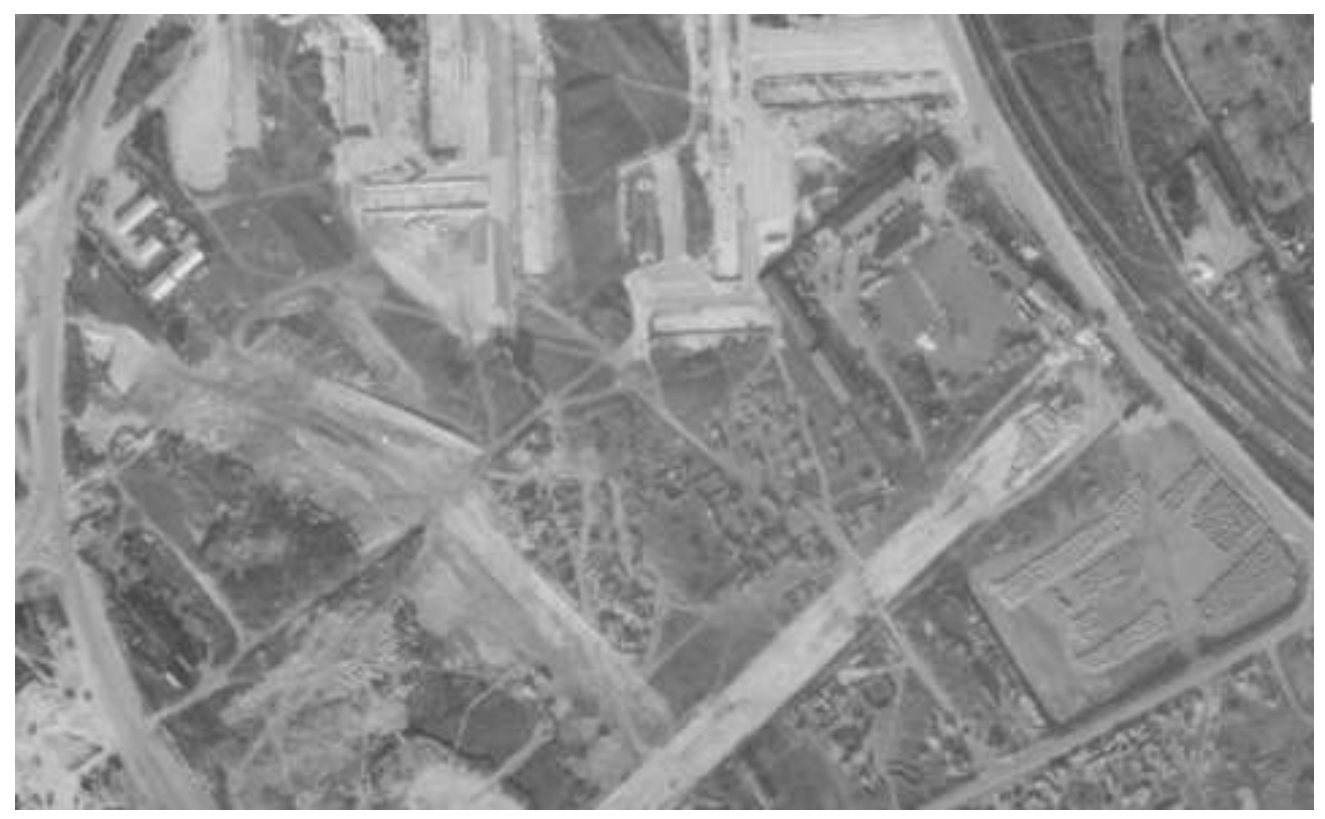

L'ensemble de la zone est en pleins travaux. Le bidonville s'est fortement agrandi et a débordé de l'autre côté de la nouvelle avenue.

(c) IGN

Quelques semaines ou mois plus tard, les promoteurs immobiliers consentent à édifier des baraquements pour loger leurs ouvriers, au plus près du chantier (figure 15). Est-ce une première réaction face au développement de ces bidonvilles ? Il s'agit peut-être de loger les ouvriers dans des conditions plus salubres et de les extraire des cabanes environ- 
nantes. On perçoit une surprenante juxtaposition de logements ouvriers entre les baraquements officiels, les taudis informels et la cité de logement social en devenir.

FIGURE 15. CONSTRUCTION DES BARAQUEMENTS POUR LES OUVRIERS DU BÂTIMENT DE LA CITÉ DES PROVINCES FRANÇAISES EN JUIN 1956

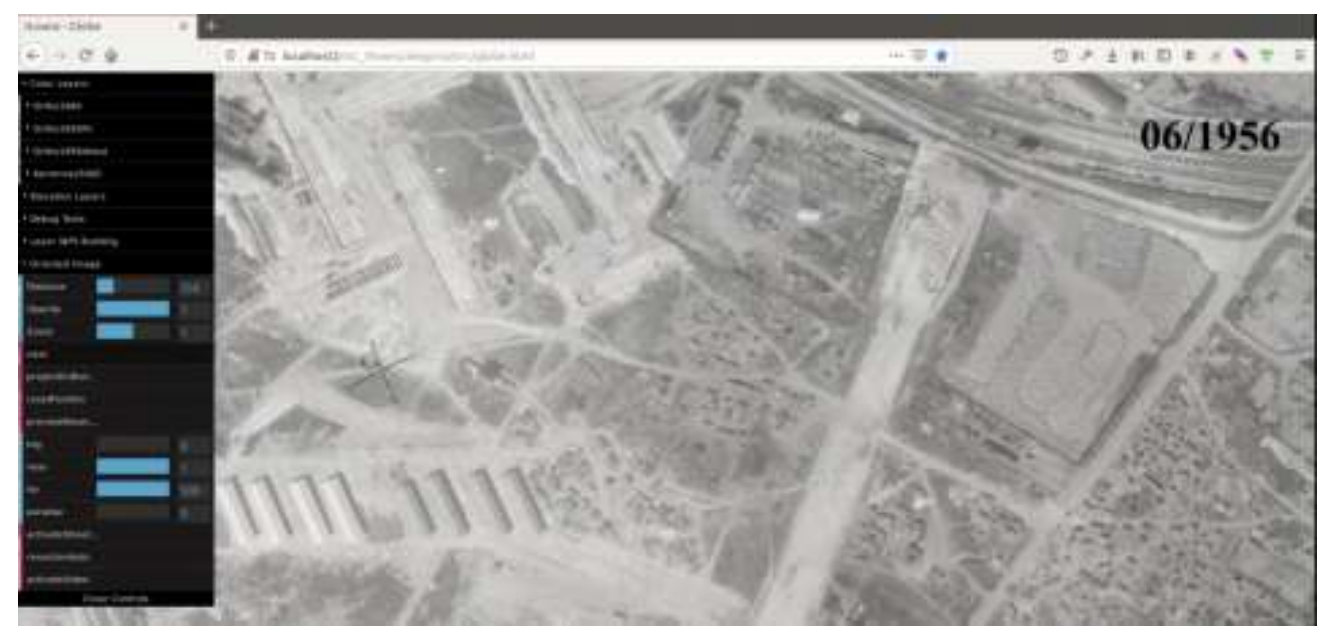

(c) IGN

La série de photographies aériennes permet ainsi de réaliser la concomitance de la formation du bidonville et des grandes opérations urbaines. Ces habitats précaires sont donc apparus très tôt, dès le début des chantiers et ont connu une très forte croissance vers le sud en direction de l'avenue Joliot-Curie, en cours d'aménagement. Ces constructions pourraient avoir servi de logements aux ouvriers du bâtiment engagés pour les grands chantiers de Nanterre, qui auraient ensuite été rejoints par d'autres travailleurs en mal de logement.

En 1961, sept ans après les premières cabanes de 1954, à l'occasion de la première mission de photographie aérienne, on est surpris de constater qu'au pied du grand ensemble, les cabanons initiaux ont disparu, et que seules subsistent les traces des chemins boueux (figure 16). Si le bidonville a été résorbé au nord, il s'est au contraire étendu, densifié, organisé et structuré au sud de l'avenue. Certains secteurs ont disparu alors que d'autres sont apparus. Le bidonville est en effet un objet mouvant, en perpétuelle reconfiguration. 


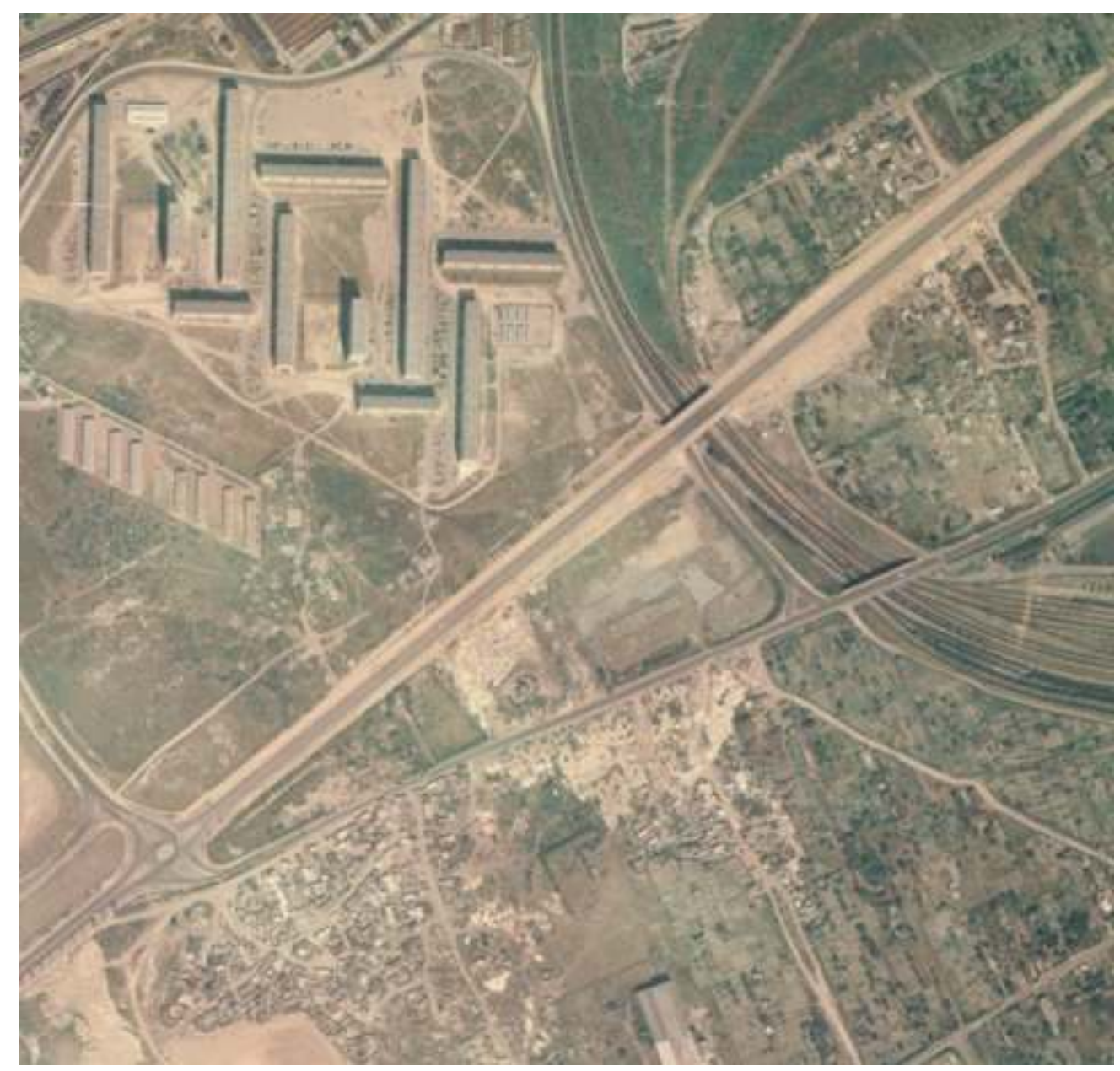

Le secteur initial du bidonville a disparu, mais l'on peut encore voir l'emprise au sol des cabanes. Au sud de l'avenue, le bidonville s'est encore densifié et structuré.

(C) IGN

L'outil de visualisation des données iTowns réunissant photographies aériennes et données du recensement offre un nouvel aperçu de la formation de l'urbain à cette époque et a permis une ébauche d'étude sur la cristallisation du bidonville de la Folie à Nanterre. L'usage des photographies obliques, peu courant chez les historiens, a conduit à une meilleure appréhension de l'espace et à une meilleure compréhension des changements urbains. Au sein de la plateforme iTowns, l'évolution des paysages est aisément visible et vient compléter les données issues des archives produites par la puissance publique. 


\section{Raconter les bidonvilles des 66 et 97 de la rue des Prés}

L'approche géosociologique développée dans le projet ALEGORIA a permis de compléter la collecte des données historiques analysées dans le projet Seine 54 (émanation du programme Archival City) par des recherches dans les archives photographiques de l'IGN et des archives départementales des Hauts-de-Seine. Ces recherches menées par une sociologue, Sylvaine Conord accompagnée d'une géographe, Lætitia Delavoipière, ont apporté de nouveaux éléments de connaissance sur l'espace des bidonvilles de la rue des Prés de Nanterre, considéré dans cette démarche sociogéographique comme un produit sociétal et non comme un objet autonome. C'est-à-dire qu'à travers l'histoire géospatiale de ces lieux, alimentée par les propos d'habitants nanterriens et l'utilisation de la plateforme iTowns, nous allions pouvoir valoriser des fonds photographiques de l'IGN et travailler sur les représentations sociales des bidonvilles à partir de témoignages sur images. Une méthodologie a été mise au point en faisant appel au recueil qualitatif d'informations orales en entretien et à la technique de la photo elicitation interview (Collier et Collier 1986 ; Conord 2007 ; Bigando 2013), c'est-à-dire la photographie comme support d'entretien libérant la parole. Le but est d'établir une évaluation des fonctions de la plateforme iTowns exprimée par des habitants de Nanterre (Camille, nouvelle directrice de l'office de tourisme de Nanterre, et Alain Bocquet, trésorier de la Société d'histoire de Nanterre [SHN]), et des ex-habitants des bidonvilles de la rue des Prés (Kader, Ahmed).

$\mathrm{Au}$ moment de l'installation des populations à Nanterre dans les années 1950, les différents bidonvilles de Nanterre n'ont pas tous la même histoire et diffèrent " par leur ampleur, par l'importance et l'origine de leurs habitants, par leur équipement "(Cohen 2011). Ainsi, les 66 et 97 rue des Prés sont les seuls à être approvisionnés en eau et en électricité (Santelli et al. 2019). Le 66 n'est fréquenté que par des familles, le 97 par des hommes seuls. À travers les témoignages de Kader et Ahmed sur leur enfance dans les bidonvilles de la rue des Prés, nous avons pu comprendre les raisons de la distinction entre le 66 et le 97 :

Les célibataires avaient une vie un peu décousue, donc il n'était pas question qu'ils se mélangent avec les familles. Parce qu'ils buvaient, ils se bagarraient... et donc ça a été fait naturellement [la séparation]. (Ahmed)

Les familles et les hommes seuls ne se mélangeant pas, les deux adresses fonctionnaient donc différemment. Par exemple, les allées du 66 étaient bétonnées, ce n'était pas le cas du 97. Les entretiens sont indispensables en sociologie pour connaître des aspects précis de la vie sociale du passé ou du présent.

Ainsi, notre corpus est composé de photographies (IGN, AD92), d'images visualisées en 3D (iTowns) et des paroles des interviewés témoins d'une réalité sociale observée. Alors que la ville de Nanterre, à l'image de ses bidonvilles, est riche historiquement, l'association des 
photographies, d'une plateforme de visualisation et des témoignages de connaisseurs grâce à l'usage de la photo elicitation ouvre une nouvelle perspective.

N'ayant qu'une série de quatre photos en 1960 présentant les 66 et 97 rue des Prés, nous avons opté pour le cliché où les deux bidonvilles sont cadrés le plus au centre possible de la photo (figure 17), permettant ainsi d'avoir un plan large sur les alentours et ainsi d'ouvrir la discussion sur les activités pratiquées autour de leur lieu de vie.

FIGURE 17. LES BIDONVILLES DE LA RUE DES PRÉS À NANTERRE DANS LES ANNÉES 1960

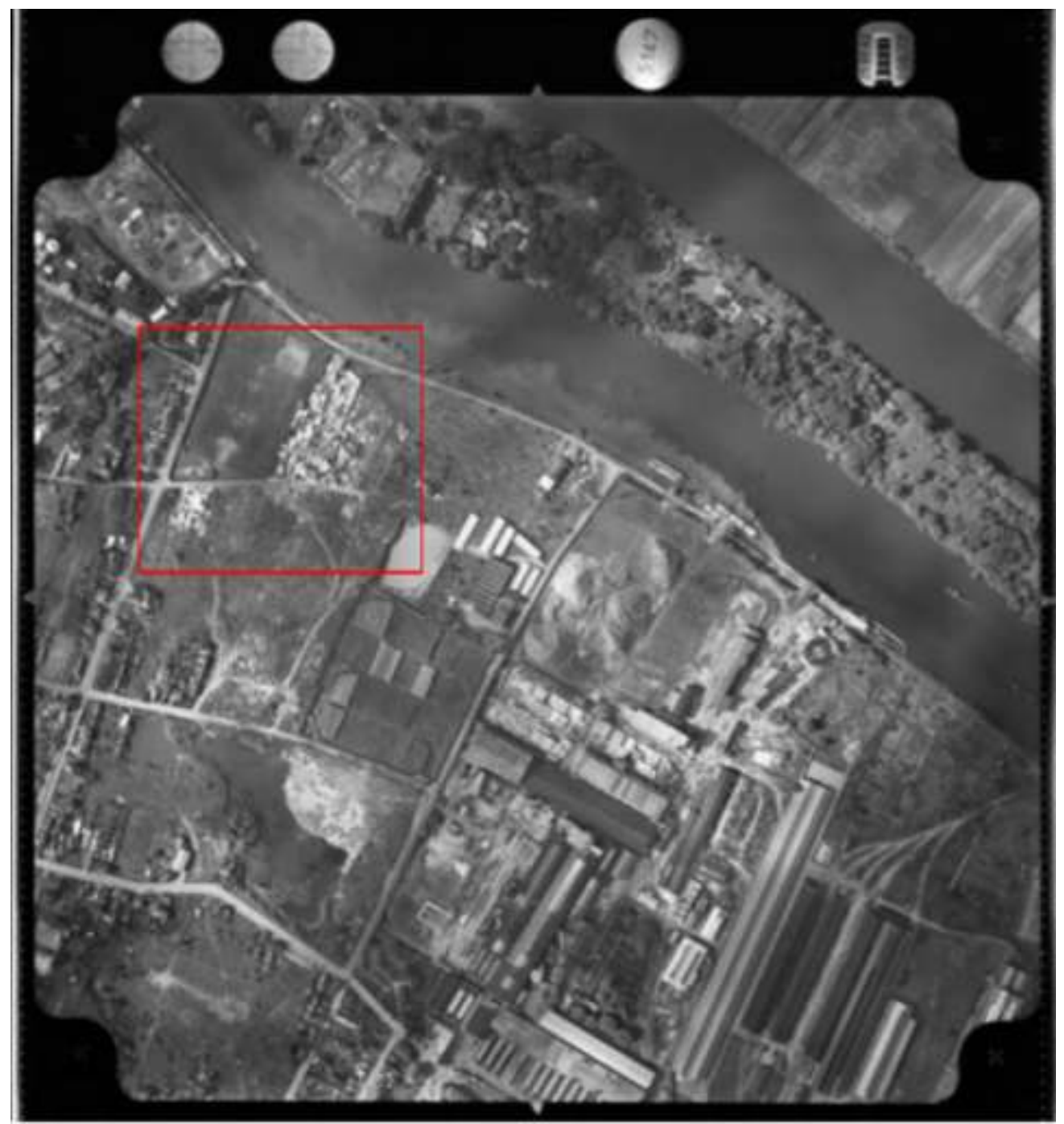

Les deux bidonvilles de la rue des Prés à Nanterre en 1960 : le 66 à gauche, le 97 à droite. (c) IGN

Contrairement à l'observation d'une photo aérienne ancienne seule, la plateforme de visualisation iTowns permet de naviguer dans un environnement tridimensionnel contemporain dans lequel on va venir intégrer des images anciennes. Ce procédé est apprécié des connaisseurs du territoire pour la mise en visibilité très nette des changements urbains au fil des années (figure 18). La plateforme iTowns a aussi été perçue comme très utile pour des visiteurs qui connaissent peu la ville et son histoire. Camille, directrice de l'office de tourisme de Nanterre, évoque le rôle du guide qui accompagne les visites de la ville. Ce guide est, selon elle, voué à disparaître pour laisser place à des balades où le visiteur est moins passif. Si la tablette requérant Internet peut poser des contraintes 
de mobilité, Camille est convaincue qu'iTowns consultable dans l'office de tourisme (situé au centre-ville) serait très attractif pour les visiteurs internes ou externes à la ville de Nanterre. Ainsi, d'anciens Nanterriens viennent régulièrement rendre visite à Camille pour retrouver le nom des rues dans lesquelles ils ont résidé, et pour admirer des photographies. N'ayant à l'office que quelques livres sur la ville, elle leur conseille de prendre contact avec la Société d'histoire de Nanterre. Mais Camille a l'intention de réaménager l'office et de l'agrémenter d'un ordinateur avec des applications pédagogiques et la plateforme iTowns, par exemple. Quand cette plateforme sera accessible au public, elle pourra éveiller la curiosité des nouveaux habitants comme des touristes.

L'objectif ce n'est pas pour les gens qui connaissent déjà le territoire, c'est vraiment pour les inconnus qui voudraient découvrir. (Camille)

Nous avons également noté à travers les résultats des entretiens quelques-unes des limites de cette plateforme, essentiellement d'ordre technique, qui pourront ainsi être prises en compte par les concepteurs de l'équipe d'ALEGORIA, afin d'améliorer les usages de cet outil performant. Alain Bocquet, connaissant très bien la ville de Nanterre et son histoire, précise que l'image proposée par la plateforme et reconstituée doit être très récente et juste.

[iTowns] ne peut pas être à jour dans une ville comme la nôtre [Nanterre] où tous les 6 mois tu as une inauguration. L'école Makeba n'y est pas. [...] On la voit en chantier [sur iTowns] alors qu'on l'a inaugurée la semaine dernière. (Alain Bocquet)

La plateforme iTowns a été présentée aux interviewés dans sa forme provisoire. Elle est en développement jusqu'en 2021, fin du projet $A L E G O$ $R I A$, et nous prolongerons d'ici là la réflexion sur l'importance de la dimension temporelle des images présentées.

Si certains bidonvilles nanterriens se résorbent au début des années 1960, les autres sont détruits en 1972. Les conditions de relogement ne sont pas les mêmes pour tous les habitants. Si une petite part des familles est hébergée en HLM, ou accède à un marché privé plus ou moins salubre, la plus grande partie, faute de ressources économiques et relationnelles, est relogée en cités de transit à Nanterre ou à Gennevilliers (cités provisoires construites pour quelques années dans l'attente des HLM). Malgré leur fonction initiale de transition, ces cités ont été maintenues longtemps en l'état. Parmi elles,

[la cité de transit] « André Doucet » qui borde le 66 rue des Prés, puis la cité "Gutenberg ", un peu plus loin, nommée par ses habitants la " cité blanche » où s'installent la plupart des familles du 66. (Kader) 


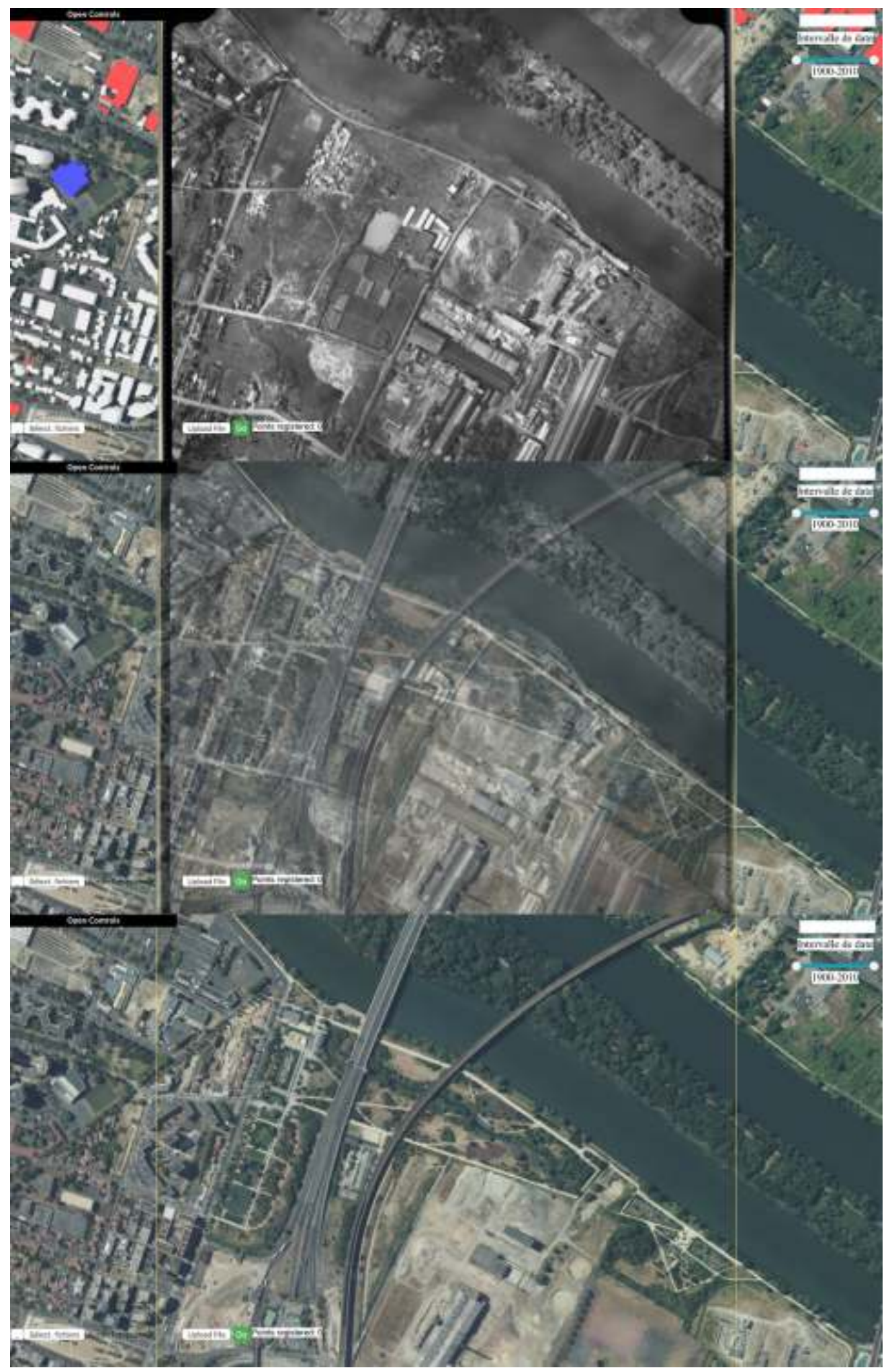

Une image de bidonville en 1960 présentée en transparence grâce à la plateforme iTowns. (c) IGN

Lire grâce à iTowns les images en transparence d'un quartier en vue aérienne prises à un moment donné dans le passé, puis rendre visibles, peu à peu, tous les changements urbanistiques de cet espace jusqu'à nos jours par la superposition de photographies récentes, offre un tout autre point de vue sur la ville, actualise les perceptions et améliore les interprétations de l'histoire d'un territoire. 


\section{Conclusion}

Dans ces deux études de cas, l'espace en trois dimensions représenté au sein de la plateforme devient une modalité privilégiée d'articulation des données. Pour les historiens, l'outil offre l'opportunité de mieux appréhender la constitution du bidonville de la Folie en croisant une étude de la morphologie sociale à une étude de la morphologie spatiale, et ce à plusieurs échelles. Pour les sociologues, cette plateforme et son outil de géoréférencement semi-automatisé sont devenus un instrument de restitution de l'histoire du territoire et une porte d'entrée afin d'étudier la mémoire de ces lieux, à partir d'une démarche de photo elicitation.

Ces premiers travaux sur la formation et les traces mémorielles des bidonvilles de Nanterre permettent, nous semble-t-il, de valider cette expérience de collaboration entre chercheurs en sciences sociales et de l'IGN. D'une part, l'outil de visualisation iTowns a fait dialoguer des documentations très disparates, offrant un nouvel outil de manipulation des données à des historiens et des sociologues. D’autre part, les retours d'expérience de cette étude de cas ont conduit l'IGN à développer de nouvelles fonctionnalités plus immersives pour un public qui ne maîtrise pas le code informatique.

Ces deux approches sociogéographique et historique de la plateforme iTowns témoignent bien des vastes possibilités d'usage de cet outil de spatialisation de données hétérogènes. Pour les chercheurs en sciences sociales, c'est une nouvelle façon d'aborder les questions urbaines en s'appropriant au mieux la réalité spatiale du passé. Au travers d'Archival City et d'ALEGORIA, les synergies interdisciplinaires ont vocation à s'approfondir et à prolonger les études réalisées sur cette plateforme en s'adressant à tous les chercheurs travaillant sur les passés de la métropole parisienne. En particulier, le recensement de 1954 offre de nombreuses pistes de recherche et Archival City souhaite mettre à disposition de tous les chercheurs ces données afin de susciter des travaux sur la métropole parisienne au milieu $\mathrm{du} \mathrm{xx}^{\mathrm{e}}$ siècle. À n'en pas douter, certaines de ces recherches chercheront à croiser cette source avec d'autres données et iTowns peut représenter une solution idoine à ces nouveaux projets. En outre, dans le cadre d'Archival City, d'autres types d'archives sont en cours de traitement et de spatialisation, tandis que l'IGN poursuit l'intégration de nouveaux fonds photographiques. Le croisement de ces nouvelles données fera certainement émerger dans les prochaines années de nouveaux sujets de recherche et de nouveaux projets en collaboration.

\section{Bibliographie}

Barros, Françoise de. 2005. « Des “Français musulmans d'Algérie” aux “immigrés”. L'importation de classifications coloniales dans les politiques du logement en France ». Actes de la recherche en sciences sociales 159 (4) : 26-53. https://doi.org/10.3917/arss.159.0026.

Barros, Françoise de. 2012. « Les bidonvilles : entre politiques coloniales et guerre d'Algérie ". Métropolitiques. https://www.metropolitiques.eu/Les-bidonvilles-entre-politiques.html. 
Barros, Françoise de et Muriel Cohen. 2019. «Entre politiques urbaines et contrôle des migrants : la décolonisation inachevée des recensements des bidonvilles en France (années 1950-années 1970) ». Histoire \& mesure XXXIV (1) : 151-184. https://doi.org/10.4000/histoiremesure.8466.

Bigando, Eva. 2013. " De l'usage de la photo elicitation interview pour appréhender les paysages du quotidien : retour sur une méthode productrice d'une réflexivité habitante ». Cybergeo : European Journal of Geography 645. https://doi.org/10.4000/cybergeo.25919.

Blanc-Chaléard, Marie-Claude. 2016. En finir avec les bidonvilles : immigration et politique du logement dans la France des Trente Glorieuses. Paris : Publications de la Sorbonne.

Brejcha, Jan et Martin Cadik. 2017. « GeoPose3K : Mountain Landscape Dataset for Camera Pose Estimation in Outdoor Environments ». Image and Vision Computing 66 : 1-14. https:// doi.org/10.1016/j.imavis.2017.05.009.

Busayarat, Chawee. 2006. "Conception d'un système de référencement spatial de photographie de relevé ». MIA Journal 2 : 89-94.

Cohen, Muriel. 2011. "Les bidonvilles de Nanterre, entre "trop plein" de mémoire et silences ». Diasporas $17: 1$-22.

Cohen, Muriel. 2012. « Regroupement familial : l'exception algérienne ». Plein droit 95 (4) : 19-22. https://www.gisti.org/spip.php?article4545.

Collier, John Jr. et Malcolm Carr. Collier. 1986. Visual Anthropology. Photography as a Research Method. Albuquerque : University of New Mexico Press.

Conord, Sylvaine, éd. 2007. " Arrêt sur images : photographie et anthropologie », Ethnologie française 37 (1).

Desrosières, Alain et Laurent Thévenot. 200o. Les Catégories socio-professionnelles. Paris : La Découverte.

Devaux, Alexandre, Mathieu Brédif et Nicolas Paparoditis. 2012. "A Web-Based 3D Mapping Application Using WebGL Allowing Interaction with Images, Point Clouds and Models ». Dans Proceedings of the 2oth ACM SIGSPATIAL International Conference on Advances in Geographic Information Systems. ACM-GIS'12, 586-588. New York : ACM. https://doi.org/10.1 $145 / 2424321.2424422$.

Escafré-Dublet, Angéline, Lionel Kesztenbaum et Patrick Simon. 2018. "La greffe coloniale en métropole. Les Français musulmans dans le recensement de 1954 ». Sociétés contemporaines 110 (2) : 35-59. https://doi.org/10.3917/soco.110.0035.

Fourcaut, Annie. 2000. La Banlieue en morceaux ? La crise des lotissements défectueux en France dans l'entre-deux-guerres. Grâne : Créaphis.

Fourcaut, Annie et Danièle Voldman. 2013. «Penser les crises du logement en Europe au $\mathrm{xx}^{\mathrm{e}}$ siècle ». Le Mouvement Social 245 (4) : 3-15. https://doi.org/10.3917/lms.245.0003.

Garcia-Fidalgo, Emilio et Alberto Ortiz. 2015. «Vision-Based Topological Mapping and Localization Methods : a Survey ». Robotics and Autonomous Systems (RAS) 64 : 1-20. https://do i.org/10.1016/j.robot.2014.11.009.

Harrach, Mouna, Alexandre Devaux et Mathieu Brédif. 2019. «Interactive Image Geolocalization in an Immersive Web Application ". ISPRS - International Archives of the Photogrammetry, Remote Sensing and Spatial Information Sciences XLII-2/W9 : 377-380. https://oi.org/ 10.5194/isprs-archives-XLII-2-W9-377-2019.

Humbert, André, Roland Courtot et Colette Renard. 2013. "Les paysages lus du ciel. De l'intérêt de la photographie aérienne oblique ". Méditerranée 120 : 111-126. https://doi.org/1 0.4000/mediterranee.6748.

Lowry, Stephanie, Niko Sünderhauf, Paul Newman, John J. Leonard, David Cox, Peter Corke et Michael J. Milford. 2016. «Visual Place Recognition : a Survey ». IEEE Transactions on Robotics (TRO) 32 (1) : 1-19. https://doi.org/10.1109/TRO.2015.2496823.

Maffre, Laurent. 2012. Demain, demain : Nanterre, bidonville de la Folie, 1962-1966. Arles : Actes Sud.

Piasco, Nathan, Désiré Sidibé, Cédric Demonceaux et Valérie Gouet-Brunet. 2018. «A Survey on Visual-Based Localization : on the Benefit of Heterogeneous Data ». Pattern Recognition 74 (février) : 90-109. https://doi.org/10.1016/j.patcog.2017.09.013.

Pinol, Jean-Luc et Maurice Garden. 2009. Atlas des Parisiens. De la Révolution à nos jours. Paris : Parigramme. 
Santelli, Serge, Isabelle Herpin, Bernard Huet et Christian de Portzamparc, éd. 2019. « La rue des Prés. Habiter un bidonville à Nanterre ». Bulletin de la Société d'histoire de Nanterre 58.

Sayad, Abdelmalek. 1995. Un Nanterre algérien, terre de bidonvilles. Paris : Éditions Autrement.

Weil, Patrick. 2005. «Le statut des musulmans en Algérie coloniale. Une nationalité française dénaturée ». Histoire de la justice 16 (1) : 93-109. https://doi.org/10.3917/rhj.016.0093.

\section{Notes}

1 Deux projets de recherche, réunissant une dizaine de chercheurs en histoire, sociologie et géomatique, ont collaboré dans le cadre de cette étude. D’une part, le projet ALEGORIA, financé par l'Agence nationale de la recherche (ANR), vise à faciliter la valorisation de fonds institutionnels iconographiques anciens à partir du développement de la plateforme iTowns. Au sein d'ALEGORIA, des sociologues et géographes ont développé une expérience de sociologie visuelle en vue de tester la plateforme sur la question de la mémoire des lieux. En parallèle, le projet Seine 54, émanation du programme Archival City, cherche à comprendre les premiers processus de métropolisation du Grand Paris au milieu du $\mathrm{xx}^{\mathrm{e}}$ siècle. Il s'agit en particulier de se pencher sur les questions d'expansion urbaine et du mal-logement à partir du croisement des données quantitatives du recensement du département de la Seine en 1954 avec le fonds de photographies aériennes de l'IGN (Institut national de l'information géographique et forestière).

2 Recensement général de la population de mai 1954. Données statistiques, sur la population et les logements des communes de la Seine. Répartition par îlot. Bibliothèque de l'Hôtel de Ville (Paris). Cote 28677 (1954)/516B 23.

3 Fonds photographiques de l'IGN et fonds photographiques des archives départementales des Hauts-de-Seine.

49 communes de banlieue ont échappé à la production de ces synthèses pour des raisons inconnues.

5 L'îlot se définit comme la plus petite partie du territoire communal, entièrement délimité par des voies communales publiques ou privées.

6 La reconnaissance optique des caractères (ou OCR, Optical Character Recognition) est un processus consistant à extraire du texte d'une image. Après OCR, le texte devient éditable sur l'ordinateur ; il n'est donc pas nécessaire de le retaper.

7 Logiciel fourni en complément du scanner de la marque Fujitsu.

8 https://www.planet.com/products/explorer/.

9 https://remonterletemps.ign.fr.

$10 \mathrm{https} / / /$ www.navigae.fr.

$11 \mathrm{http} / / /$ www.inventerlegrandparis.fr.

12 https://www.mapillary.com.

13 https://www.navilium.com.

14 https://www.historypin.org.

15 http://archimedial.eu.

16 http://www.aioli.cloud.

17 https://smapshot.heig-vd.ch.

18 Le MNT est un ensemble de points 3D contenant les données d'altitude d'un territoire sous la forme de semis de points irréguliers ou de grille de points à maille carrée ou triangulée qui représente le sol. Le MNS est similaire à un MNT, mais il contient les données d'altitude non seulement du sol, mais aussi du sursol (bâti et végétation).

19 Sont considérés en état de surpopulation critique, les logements où le nombre de personnes du ménage représente le double du nombre de pièces, à l'exception des logements avec une seule pièce où la jauge est fixée à trois résidents.

20 Le regroupement familial prend son essor à la fin des années 1960 et pendant les années 1970 à la suite du boom économique. Le décret de 1976 entérine des pratiques courantes d'immigration familiale (Cohen 2012). 
21 Ils sont enregistrés dans les catégories types de ménages « autres ", distincts des « ménages ordinaires ".

\section{Auteurs}

\section{Paul Lecat}

EA 3350 ACP, université Gustave-Eiffel, Marne-la-Vallée, France

Doctorant et ingénieur d'études au sein du projet Archival City, Paul Lecat est spécialiste des périphéries populaires de la métropole parisienne aux $\mathrm{XIx}^{\mathrm{e}}$ et $\mathrm{Xx}^{\mathrm{e}}$ siècles.

ORCID 0000-0003-0588-0900

paul.lecat@univ-eiffel.fr

\section{Émile Blettery}

EA 3350 ACP, université Gustave-Eiffel, Marne-la-Vallée, France

Ingénieur d'études en géomatique à l'ENSG, Émile Blettery est membre des projets Archival City et ANR ALEGORIA. Il conduit ses recherches sur la visualisation Web de données anciennes à l'université Gustave-Eiffel dans les laboratoires ACP et LASTIG.

emile.blettery@gmail.com

\section{Lætitia Delavoipière}

Université Sorbonne-Paris-Nord, Villetaneuse, France

Lætitia Delavoipière est étudiante en master 2 Géographie et aménagement du territoire, spécialité Territoires et développement durable à l'université Sorbonne-Paris-Nord. Elle a participé au projet ANR ALEGORIA dans le cadre d'un stage de trois mois au laboratoire LAVUE.

laetitia.delavoipiere@laposte.net

\section{Frédéric Saly-Giocanti}

EA 3350 ACP, université Gustave-Eiffel, Marne-la-Vallée, France

Frédéric Saly-Giocanti est maître de conférences en histoire contemporaine. Il travaille sur l'histoire urbaine de l'Allemagne et de la France. Il est spécialiste des sources statistiques et de l'usage des méthodes quantitatives et cartographiques en histoire.

ORCID 0000-0002-0260-3521

frederic.saly-giocanti@u-pem.fr

\section{Sylvaine Conord}

UMR 7218 LAVUE, université Paris-Nanterre, Nanterre, France

Sylvaine Conord est maîtresse de conférences en sociologie, spécialisée en sociologie visuelle urbaine et membre du projet ANR ALEGORIA.

sconord@parisnanterre.fr

\section{Valérie Gouet-Brunet}

UMR LASTIG, IGN, Saint-Mandé, France

Valérie Gouet-Brunet est directrice de recherche au ministère de la Transition écologique et solidaire (MTES), en poste à l'IGN dans le laboratoire LASTIG. Ses recherches en sciences et technologies de l'information et de la communication portent sur l'indexation multimodale d'images à large échelle appliquée à la valorisation du patrimoine culturel et naturel. Elle est responsable du projet ANR ALEGORIA et membre du projet Archival City et du comité de pilotage de l'association européenne Time Machine.

ORCID 0000-0003-3666-5146

valerie.gouet@ign.fr

\section{Alexandre Devaux}

UMR LASTIG, IGN, Saint-Mandé, France

Alexandre Devaux est ingénieur de recherche en géovisualisation, spécialisé dans les méthodes de rendu $3 \mathrm{D}$.

alexandre.devaux@ign.fr 


\section{Mathieu Brédif}

UMR LASTIG, IGN, Saint-Mandé, France

Mathieu Brédif est ingénieur des mines, spécialisé en géovisualisation et dans les méthodes de rendu $3 \mathrm{D}$.

mathieu.bredif@ign.fr

\section{Frédéric Moret}

EA 3350 ACP, université Gustave-Eiffel, Marne-la-Vallée, France

Frédéric Moret est professeur d'histoire contemporaine. Il est spécialiste de l'histoire urbaine en France et en Angleterre. Membre du programme de recherche Archival City, il y dirige le projet Seine 54 .

frederic.moret@univ-eiffel.fr

\section{Droits d'auteur}

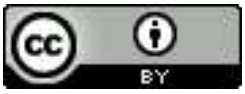

Les contenus de la revue Humanités numériques sont mis à disposition selon les termes de la Licence Creative Commons Attribution 4.0 International. 